\title{
Complutum
}

ISSN: 1131-6993

\section{Arte Rupestre y Morfometría Geométrica. Las manifestaciones rupestres de camélidos en el sur de Pozuelos (Puna de Jujuy, Argentina), durante los Desarrollos Regionales (900-1430 DC)}

\author{
Silvina Rodríguez Curletto ${ }^{1}$ y Carlos Angiorama ${ }^{2}$
}

Recibido: 29 de marzo de 2019 / Aceptado: 4 de noviembre de 2019

Resumen. La figura del camélido constituye una de las manifestaciones rupestres más abundante en el Noroeste Argentino durante el Período de Desarrollos Regionales (ca. 900-1430 DC). El objetivo principal de este trabajo es abordar la variación morfológica cuantitativa de la figura del camélido en el arte rupestre del sur de la cuenca de Pozuelos (Puna de Jujuy, Argentina) para el período referido. Para ello, se analiza una muestra conformada por 330 figuras de camélidos, ejecutados mediante las técnicas de pintura, grabado y pictograbado, plasmados en diez paisajes rupestres del sur de Pozuelos. Para la descripción cuantitativa de la forma se implementaron técnicas de Morfometría Geométrica y métodos estadísticos multivariados. Se aplicaron el método de superposición (Procrustes), Análisis de Componentes Principales, Procrustes ANOVA, Regresión Multivariada, Modularidad, Mínimos Cuadrados Parciales, Análisis de Variantes Canónicas, y Análisis de la Función Discriminante. Los resultados alcanzados constituyen una primera aproximación desde la Morfometría Geométrica para al estudio de la figura del camélido en el arte rupestre del sur de Pozuelos. Los estudios cualitativos previos sobre el estilo, paisajes y contextos arqueológicos del arte rupestre, se articulan con los resultados de la morfología cuantitativa. Esto permite corroborar la presencia de alometría, modularidad, integración morfológica y, diferencias y distancias morfológicas significativas en las figuras del camélido vinculadas a diferentes paisajes rupestres y a las Modalidades Estilísticas definidas previamente para nuestra microrregión.

Palabras clave: Morfometría geométrica; camélidos; arte rupestre; Puna de Jujuy; Argentina.

[en] Rock Art and Geometric Morphometry. The cave manifestations of camelids in the south of Pozuelos (Puna de Jujuy, Argentina), during the Regional Developments (900$1430 \mathrm{AD})$

Abstract. The figure of the camelid constitutes one of the most abundant rock art manifestations in the Argentine Northwest during the Period of Regional Developments (900-1430 AD). The main objective of this work is to approach the quantitative morphological variation of the camelid figure in the south rock art of the Pozuelos basin (Puna de Jujuy, Argentina) for the referred period. For this purpose, a sample consisting of 330 camelid figures, executed by means of painting, engraving and picto-engraving techniques, present in ten rock art landscapes of the south of Pozuelos, is analized. Geometric Morphometry techniques and multivariate statistical methods were implemented for the quantitative description of the form. The method of superposition (Procrustes), Analysis of Principal Components, Procrustes ANOVA, Multivariate Regression, Modularity, Partial Least Squares, Analysis of Canonical Variants, and Analysis of the Discriminating Function were applied. The results obtained constitute a first approach from the Geometric Morphometry to the camelid figure study in the rock art of the south of Pozuelos. The previous qualitative studies on the style, landscapes and archaeological contexts of rock art, are articulated with the results of quantitative morphology. This allow corroborating the presence of allometry, modularity and morphological integration and significant morphological differences and distances betwen camelid figures linked to the rock art landscapes and the Stylistic Modalities previously defined for our microregion.

Keywords: Geometric morphometry; camelids; rock art; Puna of Jujuy; Argentina.

\footnotetext{
Instituto de Ciencias de la Tierra, Biodiversidad y Sustentabilidad Ambiental (ICBIA). Universidad Nacional de Río Cuarto (UNRC)- Consejo Nacional de Investigaciones Científicas y Técnicas (CONICET). Instituto de Arqueología y Museo (FCNeIML- UNT. Ruta 36, Km. 602 (X5804BYA), Río Cuarto, Córdoba, Argentina. E-mail: silvina.curletto@gmail.com

2 Instituto Superior de Estudios Sociales (ISES), Consejo Nacional de Investigaciones Científicas y Técnicas (CONICET). Instituto de Arqueología y Museo (FCNeIML- UNT). San Martín 1545 (4000), San Miguel de Tucumán, Argentina. E-mail: carlosangiorama@gmail.com
} 
Sumario. 1. Introducción. 2. La figura del camélido en el arte rupestre del sur de Pozuelos. 3. Introducción a la Morfometría Geométrica de la figura del camélido. 4. Materiales y Métodos. 4.1. Definición de la muestra. 4.2. Preparación de la muestra estudiada. 4.3. Procedimientos y Análisis estadísticos aplicados. 5. Análisis de los Resultados. 5.1. Las manifestaciones rupestres de camélidos y sus contextos arqueológicos. 5.2. Morfología cuantitativa de los camélidos. 5.2.1. Análisis de componentes Principales (ACP). 5.2.2. ANOVA de Procrustes. 5.2.3. Regresión Multivariada. 5.2.4. Modularidad. 5.2.5. Mínimos Cuadrados Parciales. 5.2.6. Análisis de Variantes Canónicas. 5.2.7. Análisis Discriminante. 6. Discusión.. 6.1. Efecto de variables cualitativas sobre la morfología cuantitativa del camélido inter modalidad estilística. Estandarización, tamaño y técnicas de ejecución. 6.2. Efecto de variables cualitativas sobre la morfología cuantitativa del camélido intra modalidad estilística. Paisaje, contexto arqueológico, diacronía, técnicas de ejecución y distancias geográficas. 7. Conclusiones. Bibliografía.

Cómo citar: Rodríguez Curletto, S. y Angiorama, C. (2019). Arte Rupestre y Morfometría Geométrica. Las manifestaciones rupestres de camélidos en el sur de Pozuelos (Puna de Jujuy, Argentina), durante los Desarrollos Regionales (900-1430 DC). Complutum, 30 (2): 415-443.

\section{Introducción}

La presencia de los camélidos en el arte rupestre de los Andes del Sur en general y en particular del Noroeste Argentino (NOA en adelante), constituye una de las manifestaciones más recurrentes en la conformación de motivos y temas en el arte rupestre de momentos de los Desarrollos Regionales (ca. 900-1430 DC). Esta situación expone la importancia transversal de estos animales en las diferentes esferas de la vida de las comunidades indígenas de estas regiones (Aschero 2000; Berenguer 2004; Troncoso 2012; Martel 2010; entre otros), y al mismo tiempo habilita desde el análisis de estas manifestaciones, la posibilidad de abordar su configuración y transposición en una gran diversidad de paisajes, soportes, temas y motivos.

Aschero (2000) plantea que el arte rupestre del área circumpuneña desde el ca. 900 a 1500 DC, registra una baja diversidad de temas con estandarización de algunos patrones en la configuración de los motivos. Tanto los cánones, patrones y variantes en la configuración de la figura del camélido, que habrían cambiado a través del tiempo, presentan para momentos tardíos una pérdida progresiva de detalles y de actitudes dinámicas, que llegan a una síntesis geométrica rectilínea de marcado estatismo.

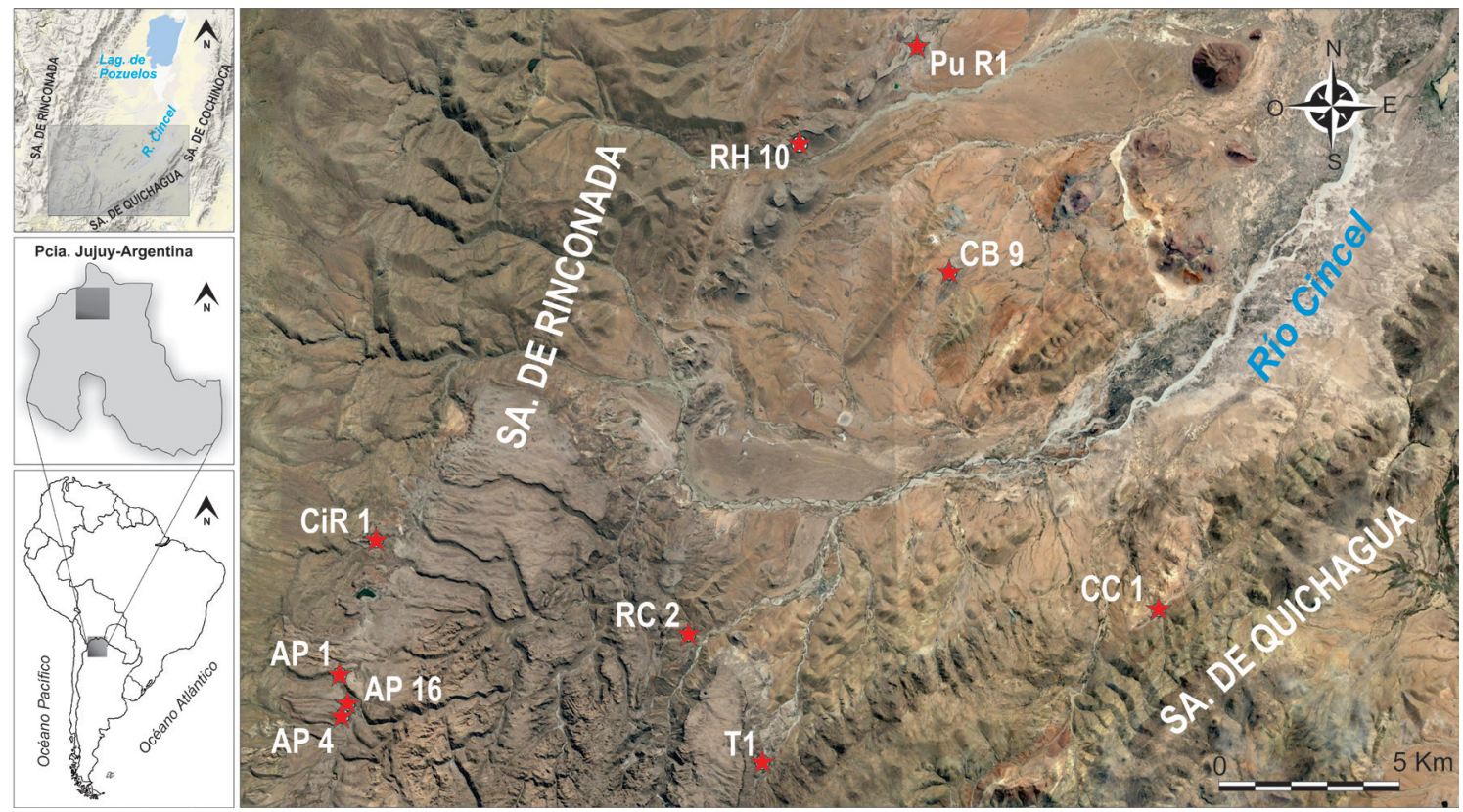

Figura 1. Mapa de ubicación de los paisajes rupestres bajo estudio. Abra Pintada 1 (AP1), Abra Pintada 4 (AP4), Abra Pintada 16 (AP16), Ciénega Rodeo 1 (CiR1), Río Candado 2 (RC2), Tabladitas 1 (T1), Casa Colorada 1 (CC1), Cerro Blanco 9 (CB9), Río Herrana 10 (RH10), Pukara de Rinconada (PuR). 
En el sur de la cuenca de Pozuelos (Fig. 1), nuestros estudios cualitativos previos nos han permitido diferenciar para momentos de los Desarrollos Regionales, dos Modalidades Estilísticas en esta microrregión, pero que presentarían un alcance regional en los Andes del Sur, ellas son la Modalidad Estilística Ciénega Rodeo (MECiR) y la Modalidad Estilística Río Herrana (MERH). En el arte rupestre de este período en general, y de estas modalidades estilísticas en particular, la figura del camélido presenta un rol preponderante con una configuración particular no solo en la definición de su morfología, sino también en las relaciones que establece con otros elementos (figuras humanas, zoomorfos, geométricos); en la conformación de diferentes motivos; así como también su disposición y emplazamiento al interior de cada espacio plástico, su relación al contexto arqueológico y de este modo en la conformación de un paisaje sociocultural en particular (Rodríguez Curletto 2014; Rodríguez Curletto y Angiorama 2016).

Estas interpretaciones previas han sido alcanzadas a través del estudio de estilos, contextos arqueológicos y paisajes del arte rupestre en cada sitio arqueológico del sur de Pozuelos. Para el estudio estilístico en general y para la composición de la figura del camélido en particular, hemos seguido los estudios previos realizados para la región circumpuneña por Aschero (2000), lo que nos permitió un primer acercamiento cualitativo a dichas configuraciones en relación a los cánones, patrones y variantes registradas dentro de cada modalidad estilística. Sin embargo, más allá de estos resultados mencionados, aún no habíamos podido abordar cómo se expresan las configuraciones de los camélidos en términos cuantitativos en las diferentes modalidades estilísticas.

En este contexto, el objetivo principal de este trabajo consiste en analizar cuantitativamente la variación morfológica y composicional (cambios y continuidades) en las manifestaciones rupestres de camélidos del sur de Pozuelos ${ }^{1}$, Puna de Jujuy (Argentina), para el período del ca. 900-1430 DC.

Para ello, en primera instancia, se aborda la variación morfológica cuantitativa de la figura del camélido a través de la aplicación de técnicas de Morfometría Geométrica (MG en adelante) basada en landmarks, y en métodos Estadísticos Multivariados (Bookstein 1995, 1996; Adams 1999; Rohlf y Marcus 1993; Slice 2007). Se analiza una muestra de 330 manifestaciones rupestres de camélidos grabados, pintados y pictograbados, emplazados en diez paisajes rupestres del sur de Pozuelos vinculados a las dos modalidades estilísticas (MERH y MECiR) definidas para momentos de los Desarrollos Regionales (Rodríguez Curletto 2014; Rodríguez Curletto y Angiorama 2016).

A partir de las coordenadas cartesianas de los hitos anatómicos considerados (landmarks), se obtuvieron las variables de tamaño y de forma a través del Análisis de Procrustes (AP) (Rohlf y Slice 1990) y luego dichas variables fueron analizadas mediante el empleo de técnicas estadísticas multivariadas con diferentes propósitos. Para reducir la dimensionalidad de los datos y explorar la diversidad y variaciones dentro de la muestra se realizó el análisis estadístico multivariado de componentes principales (ACP) (Bookstein 1996; Mitteroecker y Gunz 2009); para cuantificar la variación observada entre modalidades estilísticas se aplicó el método de Procrustes ANOVA(Klingenberg y McIntyre 1998); para abordar la covariación entre las diferentes variables, se aplicó la evaluación de la hipótesis de Modularidad (Klingenberg 2009), el método de Mínimos Cuadrados Parciales (PLS en inglés) (Bookstein et al. 2003; Klingenberg 2009) y la técnica de Regresión Multivariada (Zelditch et al. 2014). Posteriormente para evaluar las diferencias y realizar comparaciones entre grupos (modalidad estilística y paisajes rupestres) se implementó el análisis de variantes canónicas (AVC) y el análisis de la función discriminante (AFD) (Zelditch et al. 2014).

En segunda instancia, con el fin de discutir la complejidad global de la figura del camélido en los diferentes paisajes socioculturales involucrados, se consideran estas variaciones morfológicas cuantitativas en relación a la información cualitativa de nuestros trabajos previos sobre la conformación (técnica, formal y de contenido) de la figura del camélido, el contexto arqueológico asociado y las propiedades de los paisajes en los que se emplaza.

Los resultados alcanzados en esta investigación constituyen un punto de partida de gran relevancia y potencial para estudios futuros, no solo para nuestra micro-región sino también como propuesta de herramienta teórico-metodológica para el estudio del arte rupestre en general.

\section{La figura del camélido en el arte rupestre del sur de Pozuelos}

Las manifestaciones rupestres analizadas en este trabajo se emplazan en el sector sur de la cuenca de Pozuelos, ubicada en la región puneña de la 
provincia de Jujuy (República Argentina). Se trata de una cuenca endorreica de $100 \mathrm{~km}$ de largo y $20 \mathrm{~km}$ de ancho, que contiene en su interior a la Laguna de Pozuelos a los 3.600 m.s.n.m, rodeada por las Sierras de Rinconada y Carahuasi al oeste, Sierra de Quichagua al sur y Sierra de Cochinoca- Escaya al este, las cuales alcanzan aproximadamente los 4.500 m.s.n.m. (Fig. 1).

En esta micro-región, que presenta un clima continental andino del tipo semidesértico, se registran evidencias de ocupaciones humanas con una profundidad temporal que alcanza los 7.000 años AP. Asimismo, para el período aquí considerado correspondiente a los Desarrollos Regionales (ca. 900-1430 DC), la diversidad y cantidad de evidencias arqueológicas sustentan la existencia de comunidades prehispánicas con una economía fundamentalmente pastoril y agrícola, con una fuerte interacción entre ellas mediante rutas de tránsito y tráfico caravanero (Angiorama 2011), y en las que habría imperado también un contexto de tensión, conflicto social y beligerancia frecuente entre grupos étnicos (Nielsen 2007; Rodríguez Curletto y Angiorama 2017). En este contexto, el arte rupestre bajo estudio se emplaza en diferentes paisajes (fig.2) vinculados tanto a prácticas agro-pastoriles como caravaneras (Rodríguez Curletto y Angiorama 2016; Rodríguez Curletto et al. 2019).
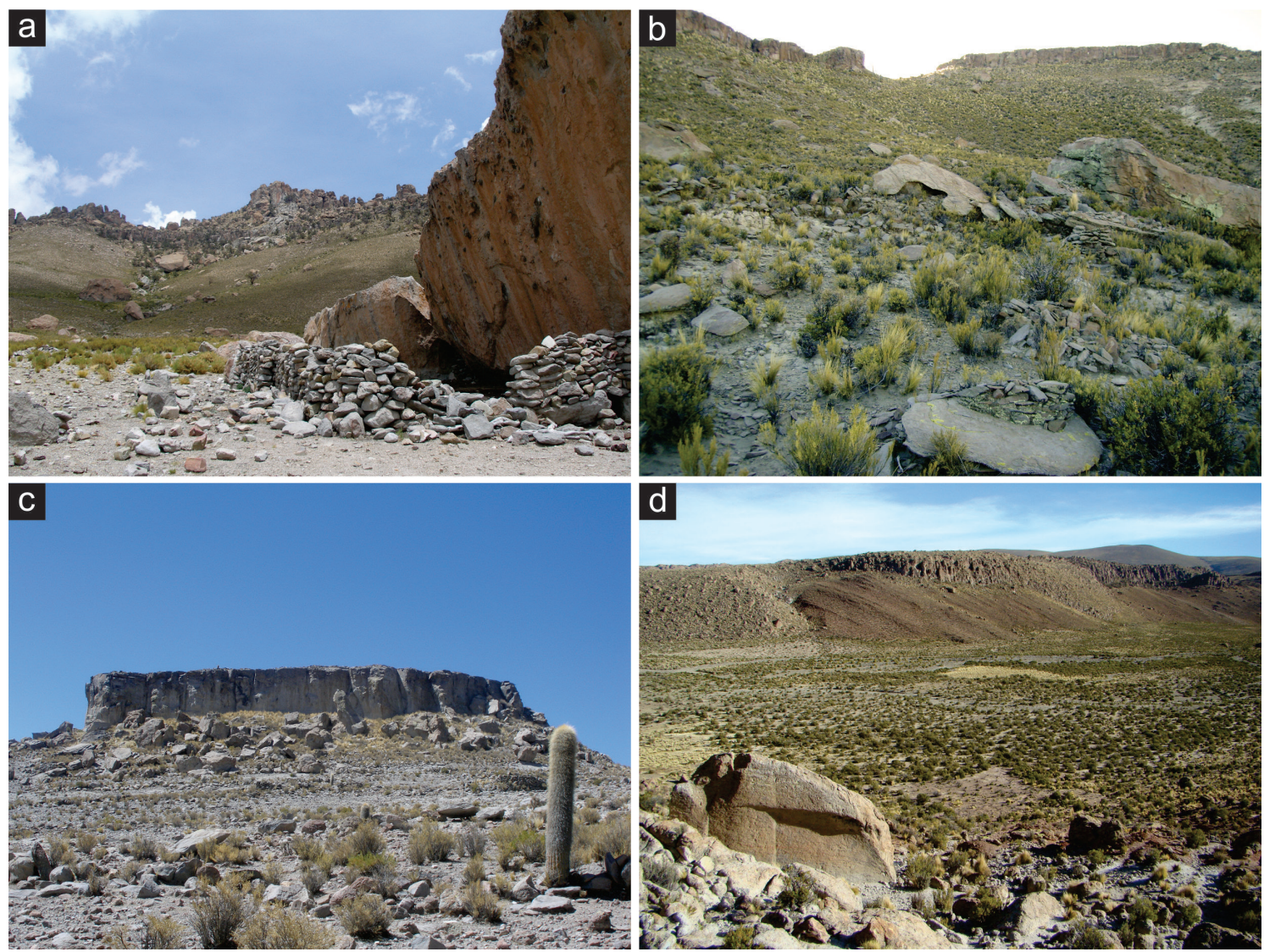

Figura 2. Paisajes rupestres del sur de Pozuelos. a. Río Candado 2; b. Río Herrana 10; c. Pukara de Rinconada; d. Abra Pintada 1

Cabe aclarar que el concepto de paisaje rupestre empleado en este trabajo se constituye como una herramienta teórica y analítica. En primera instancia, el paisaje es entendido como un fenómeno ligado a la percepción humana que expresa una significación y una lógica singular de aquellos que habitaron y modificaron sus lugares (Ingold 2000; Tilley 2004). En segunda instancia, esos lugares diferenciados del espacio abstracto, conforman al paisaje rupestre como loci que aglutina un conjunto de unidades topográficas ${ }^{2}$ (UT) con manifestaciones rupestres, vinculadas todas ellas a un contexto arqueológico y socio-cultural particular. 
El estudio cualitativo (estilo, paisaje y contexto arqueológico) del arte rupestre local, nos permitió definir dos modalidades estilísticas que se encuentran interactuando de diferentes modos; la Modalidad Estilística Ciénega Rodeo (MECiR) y la Modalidad Estilística Río Herrana (MERH) (Rodríguez Curletto 2014; Rodríguez Curletto y Angiorama 2016). Ambas modalidades jugaron un rol importante en relación a las lógicas y rituales, tanto del tráfico caravanero, de las prácticas agrícolas, así como también de la movilidad trashumante de las prácticas pastoriles (Rodríguez Curletto et al. 2019).

En la región del NOA, la figura del camélido presenta cierta recurrencia en algunos rasgos particulares que permiten su caracteri- zación morfológica y su asociación a cánones, patrones y variantes definidas por Aschero (2000). Para nuestra microrregión de Pozuelos, y en el contexto de las modalidades estilísticas MERH y MECiR (Fig. 3), hemos identificado para la figura del camélido el canon $\mathrm{Ca}-\mathrm{H}$, los patrones $\mathrm{H} 2$ y $\mathrm{H} 3$, y las variantes $\mathrm{H} 2 \mathrm{a}$ y H2b (sensu Aschero 2000). Los camélidos de la MERH se encuentran ejecutados mediante la técnica grabado (picado en surco irregular), mientras que en la MECiR está ejecutados mediante tres técnicas diferentes: la pintura (colores negro, rojo y blanco), el grabado (picado en surco, plano y en punto muy regular con diferentes grados de abrasión interna); y también del pictograbado, combinando las dos técnicas anteriores.
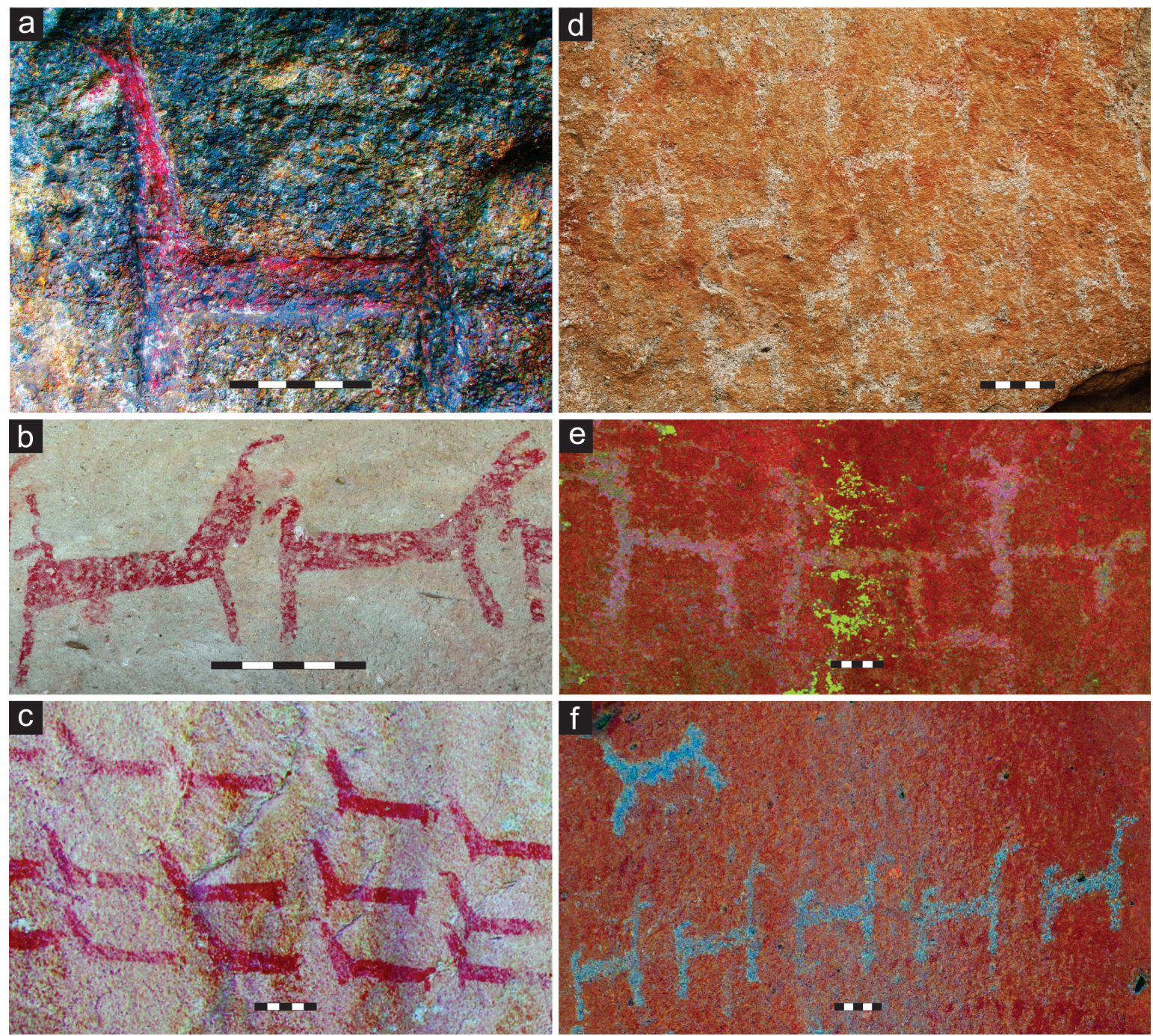

Figura 3. Manifestaciones rupestres de camélidos del sur de Pozuelos. a. Río Candado 2 (MECiR), Pukara de Rinconada (MECiR); c. Ciénega Rodeo 1 (MECiR); d. Río Candado 2 (MERH); e y f. Pukara de Rinconada (MERH). 
Las diferentes variantes de la figura del camélido conforman en los contextos caravaneros, motivos de caravanas con o sin agente guía, que se articulan de diferentes modos a las rutas de tráfico prehispánico. Por otra parte, en los contextos agro-pastoriles esta figura integra, motivos de camélidos agrupados, en rebaños, enfrentados, con crías, en corrales y vinculados también a diversos patrones de la figura humana (Rodríguez Curletto et al. 2019).

Si bien el estudio cualitativo nos ha permitido un valioso primer acercamiento a la figura de los camélidos en nuestra microrregión, extendiendo incluso dichas identificaciones a nivel regional, aún no hemos podido afrontar el cambio morfológico de modo continuo y gráfico, y así abordarlo de manera cuantitativa e independiente de los cambios de tamaño que vemos entre modalidades estilísticas y paisajes rupestres. Este motivo, nos llevó a abordar la variación morfológica cuantitativa de dicha figura a través de la Morfometría Geométrica.

\section{Introducción a la Morfometría Geométrica de la figura del camélido}

En los últimos años, varios trabajos sobre diferentes objetos de estudio destacan el potencial en el uso de las técnicas de MG que permiten justamente abordar el cambio morfológico y métrico a través de la aplicación de análisis multivariados y test de hipótesis (Torcida y Pérez 2012; Benítez y Puschel 2014; Perez et al. 2009; Cardillo 2010; Nelson et al. 2017; entre otros). Asimismo, para nuestra región son muy escasos los trabajos que utilizan estas técnicas para abordar el estudio del arte rupestre (Charlin y Hernández Llosas 2016).

Los estudios de MG son relevantes para este caso porque no se limitan al análisis de datos lineales de la figura del camélido (alto y ancho, por ejemplo) que son medidos directamente sobre el panel en el relevamiento de campo, y suelen ser los datos principales con los que contamos de las manifestaciones rupestres; sino que utiliza los datos geométricos de la forma del camélido en sí mismo. Esto permite el análisis de los datos en forma de matrices de coordenadas geométricas de la morfología de la figura, la obtención de las variables de la forma y de este modo aplicar diferentes análisis estadísticos para evaluar como se da la covaración y las diferencias de las formas registradas.

En este trabajo se presenta la utilización de métodos de MG basados en landmarks (Book- stein 1995, 1996; Adams 1999; Rohlf y Marcus 1993; Slice 2007; Zelditch et al. 2014), que consiste en analizar los cambios morfológicos de la figura del camélido tomando como base las posiciones relativas de un conjunto de puntos morfométricos homólogos ${ }^{3}$. De esta manera, el landmark se conforma como un punto definido, en nuestro caso en el plano 2D, que refiere a la ubicación concreta de un rasgo específico (aquí caracteres anatómicos) que tiene presencia equivalente en el resto de las figuras de camélidos seleccionadas. Para la definición del conjunto de hitos se consideraron los criterios de consistencia de la posición relativa, cobertura adecuada de la forma (de acuerdo al objetivo de este trabajo), repetividad, coplanaridad y el ya mencionado criterio de homología (Zelditch et al. 2014). Debido a que el método de landmarks solo puede comparar rasgos que estén presentes en todas las figuras de camélidos seleccionados, constituye una limitación no poder dar cuenta de la presencia de rasgos nuevos como puede ser la presencia de jabot o enflorados por ejemplo, para lo cual se sugiere el análisis de contornos mediante la incorporación de semilandmarks (Bookstein 1996-1997; Gunz y Mitteroecker 2013). Sin embargo, de acuerdo a los objetivos de este trabajo, el método de landmarks resulta pertinente para esta primera aproximación a la morfología cuantitativa de la figura del camélido en las diferentes modalidades estilísticas y paisajes rupestres del sur de Pozuelos.

A partir de la obtención de las coordenadas cartesianas de los landmarks, se consiguen las variables de tamaño y de forma a través del Análisis de Procrustes (AP) (Rohlf y Slice 1990) y luego dichas variables se analizan mediante el empleo de técnicas estadísticas multivariadas con diferentes propósitos, las cuales son detalladas a continuación en el apartado 4.3 (Klingenberg 2009, 2011; Bookstein 1995, 1996; Goodall 1991; Zelditch et al. 2014; Slice 2007; Klingenberg y McIntyre 1998; Bookstein et al. 2003; Charlin y Hernández Llosas 2016; Zelditch et al. 2014).

\section{Materiales y Métodos}

\subsection{Definición de la muestra}

La muestra analizada se encuentra conformada por un total de 330 figuras de camélidos (elementos) $)^{4}$, ejecutados mediante las técnicas de pintura, 
grabado y pictograbado, plasmados en 52 unidades topográficas (UT) distribuidas en 10 paisajes rupestres del sur de Pozuelos, ellos son: Abra Pintada 1 (AP1), Abra Pintada 4 (AP4), Abra Pintada 16 (AP16), Ciénega Rodeo 1 (CiR1), Río Candado 2 (RC2), Tabladitas 1 (T1), Casa Colorada 1 (CC1), Cerro Blanco 9 (CB9), Río Herrana 10 (RH10), Pukara de Rinconada (PuR).
Los estudios estilísticos realizados desde nuestros trabajos previos, nos permitieron asignar a priori estas manifestaciones de camélidos en relación a las dos modalidades estilísticas definidas para la microrregión: MECiR y MERH (Rodríguez Curletto 2014; Rodríguez Curletto y Angiorama 2016) (Tabla 1).

Tabla 1. Manifestaciones de camélidos (elementos) de la MERH y MECiR en los paisajes rupestres del sur de Pozuelos considerados en este estudio.

\begin{tabular}{|l|c|c|c|c|c|}
\hline \multirow{2}{*}{ Paisaje rupestre } & \multirow{2}{*}{ ID } & \multirow{2}{*}{$\begin{array}{c}\text { UT } \\
\text { (N) }\end{array}$} & $\begin{array}{c}\text { MERH } \\
\text { (n) }\end{array}$ & $\begin{array}{c}\text { MECiR } \\
\text { (n) }\end{array}$ & (N) \\
\hline Abra Pintada 1 & & & 0 & 6 & 6 \\
\hline Abra Pintada 4 & AP1 & 1 & 0 & 11 & 11 \\
\hline Abra Pintada 16 & AP4 & 1 & 0 & 12 & 12 \\
\hline Ciénega Rodeo 1 & AP16 & 1 & 0 & 21 & 21 \\
\hline Río Candado 2 & CR1 & 5 & 4 & 43 & 87 \\
\hline Tabladitas 1 & RC2 & 8 & 44 & 12 & 12 \\
\hline Casa Colorada 1 & T1 & 4 & 0 & 21 & 21 \\
\hline Pukara de Rinconada & CC1 & 3 & 0 & 42 & 115 \\
\hline Cerro Blanco 9 & PuR & 21 & 73 & 0 & 3 \\
\hline Río Herrana 10 & CB9 & 2 & 3 & 0 & 42 \\
\hline Totales & RH10 & 6 & 42 & 168 & 330 \\
\hline
\end{tabular}

\subsection{Preparación de la muestra estudiada}

Siguiendo el protocolo de trabajo propuesto por Charlin y Hernández Llosas (2016), se prepararon las imágenes digitales con la misma resolución $(d p i)$, orientación e inclinación para cada unidad de análisis correspondiente a la figura del camélido, considerado aquí como elemento. Se decidió como norma, orientar las figuras de los camélidos con vista hacia la izquierda y corregir la inclinación de cada elemento, siempre sin alterar su configuración original. Se incluyó una escala gráfica en cada imagen con el fin de escalar cada elemento analizado mediante el programa tps Digitaizing 2 (Rolhf 2013). Luego, se realizó la nomenclatura de cada imagen considerando nuestra categorización previa de las figuras del camélido según las modalidades estilísticas MERH y MECiR (Rodríguez Curletto 2014; Rodríguez Curletto y Angiorama 2016), seguidas por el nombre de los paisajes rupestres en los que fueron registrados para facilitar posteriormente la identificación de cada elemento en relación a estas dos va- riables (estilo y localización). Se generó el acopio de imágenes preparadas para el análisis mediante el programa tps Utility (Rohlf 2008), luego se realizó la digitalización de la escala y de los landmarks de cada uno de los elementos mediante el programa tps Digitizing 2 (Rohlf 2013).

Con el objeto de estandarizar la nomenclatura de las regiones anatómicas de los camélidos, en la figura 4a se presentan los puntos morfométricos seleccionados para describir dicha figura a partir de regiones zoométricas estándar (Quispe Coaquira et al. 2015) junto a otros rasgos anatómicos que hemos considerado importantes para la caracterización de los camélidos en el arte rupestre ${ }^{5}$. De esta manera, se define para cada imagen con la figura del camélido, las coordenadas cartesianas de cada hito morfológico a partir de la digitalización del conjunto de puntos morfométricos previamente pautados (Fig. 4). De acuerdo a los objetivos de este trabajo, se definieron 21 landmarks para cada uno de los 330 elementos, lo cual conforma un total de 6.930 puntos morfométricos digitalizados (Tabla 2). 

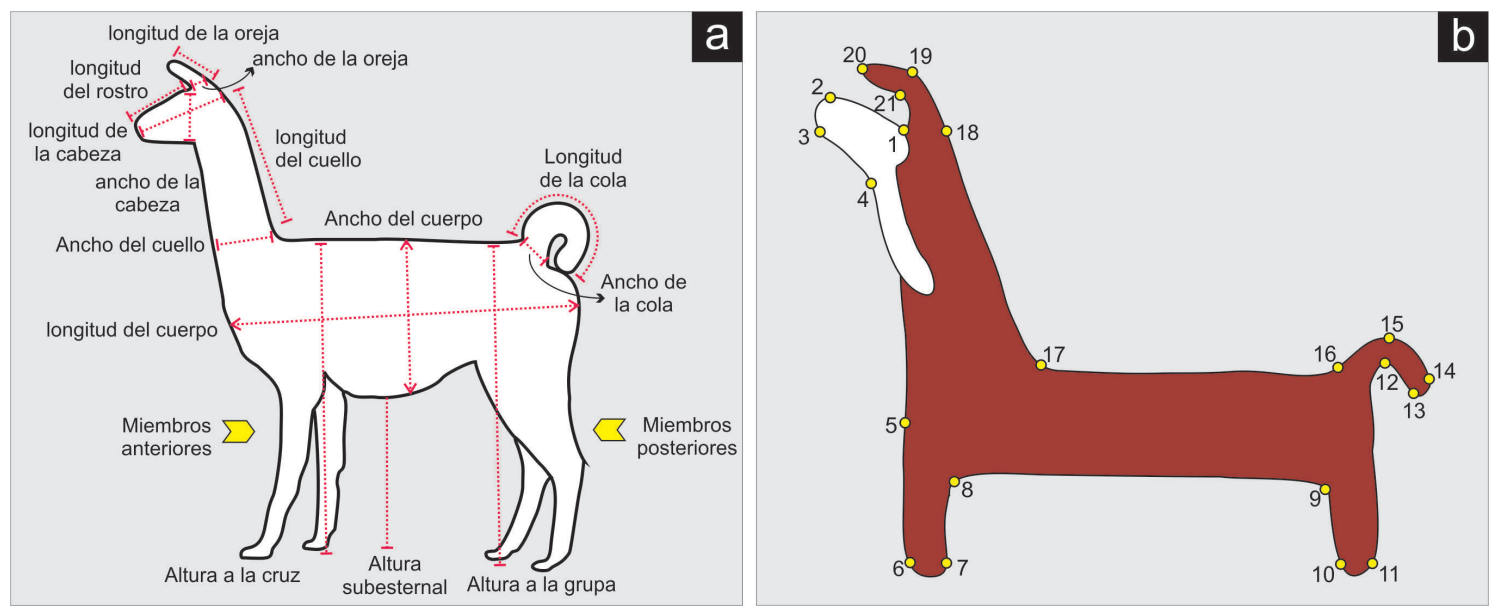

Figura 4. a. Regiones zoométricas y rasgos anatómicos relevantes en la caracterización de los camélidos;

b. Hitos o landmarks definidos para el análisis morfogeométrico de la figura del camélido (camélido de referencia del paisaje rupestre Ciénega Rodeo 1).

Tabla 2. Caracterización de la posición anatómica sobre la figura del camélido de los 21 landmarks considerados.

\begin{tabular}{|l|l|}
\hline Landmark $\mathbf{n}^{\mathbf{0}}$ & \multicolumn{1}{c|}{ Posición anatómica en la figura del camélido } \\
\hline 1 & Unión de la cara con base de la oreja \\
\hline 2 & Extremo distal de la cara (parte incisiva en maxilar superior) \\
\hline 3 & Extremo distal de la cara (parte incisiva del cuerpo mandibular) \\
\hline 4 & Unión de la cara (sector inferior del ángulo mandibular) con el cuello \\
\hline 5 & Sector medio de cintura escapular \\
\hline 6 & Extremo distal de miembro anterior (dorsal) \\
\hline 7 & Extremo distal de miembro anterior (plantar) \\
\hline 8 & Unión miembro anterior con el tórax \\
\hline 9 & Unión miembro posterior con el abdomen \\
\hline 10 & Extremo distal de miembro posterior (dorsal) \\
\hline 11 & Extremo distal de miembro posterior (plantar) \\
\hline 12 & Unión de cintura pélvica y vértebras caudales \\
\hline 13 & Extremo distal inferior de vértebras caudales \\
\hline 14 & Extremo distal superior de vértebras caudales \\
\hline 15 & Sector medio superior de vértebras caudales \\
\hline 16 & Unión de vértebras sacras y caudales \\
\hline 17 & Unión de vértebras cervicales y torácicas \\
\hline 18 & Unión base del cráneo, inicio de columna cervical \\
\hline 19 & Sector medio exterior de la oreja \\
\hline 20 & Extremo distal de la oreja \\
\hline 21 & Sector medio interior de la oreja \\
\hline
\end{tabular}

\subsection{Procedimientos y Análisis estadísticos aplicados}

Para responder a las diferentes preguntas sobre cuáles y cómo ocurren las diferencias morfológicas y la variabilidad en los "modos de hacer" las manifestaciones rupestres de camélidos entre modalidades estilísticas y en los diferentes paisajes rupestres, se llevaron a cabo sobre los datos, diversos procesamientos y análisis estadísticos multivariados utilizando el paquete MorphoJ. 
En primera instancia, se realizó la importación desde el Programa MorphoJ (Klingenberg 2011) del archivo tps generado previamente conteniendo todos los hitos digitalizados (6930 landmarks) a partir de los cuales se realizó el análisis generalizado de Procrustes (Rohlf y Slice 1990; Bookstein 1995; Goodall 1991). Debido a la superimposición de los cuadrados mínimos de landmarks (Slice 2007) se generan las "coordenadas de forma de Procrustes" que implica la remoción de los efectos de rotación, traslación y escala en las configuraciones de los landmarks, y por otra parte, el promedio de Procrustes que genera la forma de referencia o consenso (tendencia central) de la muestra total (Bookstein 1996).

Para reducir la dimensionalidad de los datos, generar nuevas variables no correlacionadas, definir los principales ejes de variaciones morfológicas, y explorar la diversidad y variaciones dentro de la muestra, se realizó el análisis estadístico multivariado de componentes principales (ACP). Para ello se utilizó la matriz de covarianzas de las coordenadas de Procrustes de todos los elementos analizados (Zelditch et al. 2014) mientras que las variaciones registradas en la configuración de la figura del camélido son evaluadas en relación a la forma consenso (tendencia central) mencionada anteriormente (Bookstein 1996). Para cuantificar la variación utilizamos el método de Procrustes ANOVA (Klingenberg y McIntyre 1998) que nos permitió evaluar la magnitud de las diferencias para el tamaño del centroide y la forma propiamente dicha (shape) al considerar los efectos producidos por los "paisajes rupestres" y las "modalidades estilísticas".

Para analizar la presencia de covariación entre las diferentes variables, se aplicaron la evaluación de la hipótesis de Modularidad (Klingenberg 2009), el método de Mínimos cuadrados parciales (PLS en inglés) (Bookstein et al. 2003; Klingenberg 2009) y la técnica de Regresión Multivariada (Zelditch et al. 2014). El análisis de la Modularidad utiliza las matrices de covariación y evalúa la existencia de unidades o módulos dentro de la configuración de la forma del camélido que presentan cierto grado de integración con una alta covariación dentro de dichos módulos y una covariación débil entre los diferentes módulos. De este modo se estima el coeficiente de RV (Escoufier 1973) para analizar la independencia relativa de los módulos hipotetizados en relación a las diferen- tes modalidades estilísticas y luego, se evalúa el nivel de integración morfológica de los mismos a través del método de mínimos cuadrados parciales (PLS) (Bookstein et al. 2003; Klingenberg 2009) que permite examinar la covariación entre los conjuntos de variables definidos y por ende, evaluar el grado de asociación entre los módulos anatómicos de las manifestaciones de camélidos. Debido a que el PLS no explica una dirección en la asociación de las variables, se utiliza la técnica de Regresión Multivariada (Zelditch et al. 2004) para conocer si los cambios observados en las formas de los camélidos, tienen algún tipo de relación con el tamaño de los mismos (alometría). De este modo, para poner a prueba la existencia de alometría se considera la variación de la "forma propiamente dicha" (shape) como un conjunto de variables dependientes (coordenadas de forma alineadas) en relación al tamaño como variable independiente (log centroid size).

Para realizar comparaciones y evaluar las diferencias entre grupos (modalidad estilística y paisajes rupestres) se implementó el análisis de variantes canónicas (AVC) y el análisis de la función discriminante (AFD) (Zelditch et al. 2014). Para ello, hemos clasificado a priori las figuras de camélidos en relación a las modalidades estilísticas definidas para nuestra microrregión (Rodríguez Curletto 2014; Rodríguez Curletto y Angiorama 2016) y también de acuerdo al paisaje rupestre en el que se encuentran plasmados (Tabla 1).

El AVC genera nuevas variables a través de un nuevo sistema de coordenadas que ayuda a describir dónde ocurren las diferencias entre las medias de estos grupos si efectivamente son diferentes, y si esto es así, de qué manera se expresan esas diferencias (Johnson 2000). Se centra en la disposición relativa de múltiples grupos. El AVC supone que la estructura de covarianza dentro de todos los grupos es la misma y por este motivo la matriz de covarianza general se utiliza en todo el AVC, incluso en el cálculo de las distancias de Mahalanobis entre pares de grupos. Por este motivo, para la comparación entre pares de paisajes rupestres por separado hemos considerado las distancias de Procrustes $\mathrm{y}$ de Mahalanobis que ofrece el AFD que considera solo las matrices de covarianza del par de grupos incluidos en una comparación. En este sentido, el análisis de la función discriminante (AFD) ${ }^{7}$, como ocurre con el AVC también examina la separación entre grupos de observaciones, pero el AFD considera solo dos grupos 
de análisis a la vez y pone énfasis en el grado de separación de los mismos y por tanto en la probabilidad de clasificaciones correctas o incorrectas de las observaciones. Por este motivo, para los pares de paisajes rupestres se considera la información del AFD sobre las diferencias de las formas de camélidos en cada uno de ellos, tomando en cuenta la distancia de Procrustes, distancia de Mahalanobis, el valor $p$ paramétrico asociado, incluyendo también los valores $\mathrm{P}$ de la prueba de permutación ${ }^{8}$. Debido a las características enunciadas, se aplicó el AVC para el análisis general de la muestra, mientras que el AFD ha sido aplicado para explicar cómo se dan las diferencias a través de comparaciones específicas sobre pares de grupos.

\section{Análisis de los Resultados}

\subsection{Las manifestaciones rupestres de camélidos y sus contextos arqueológicos}

Los diez paisajes rupestres considerados en este trabajo emplazados en el sur de la cuenca de Pozuelos, han sido agrupados en tres sectores: 1) límite suroeste en las Sierras de Rinconada-Carahuasi (AP1, AP4, AP16, CiR1), 2) sector meridional en la Sierra de Quichagua (RC2, T1 y CCo1) y, 3) sector septentrional del sur de la cuenca que corresponde al fondo de bolsón (PuR, CB9, RH10). Cada uno de estos sectores presenta fisiografias diferentes que se mencionan en la tabla 3 , junto a las propiedades de las manifestaciones rupestres en general, el emplazamiento y los contextos arqueológicos asociados a cada uno de los paisajes rupestres (Rodríguez Curletto 2014).

El paisaje Rupestre $\mathrm{CC} 1$ presenta ambas modalidades estilísticas (MERH y MECiR), sin embargo para este estudio solo han sido consideradas los elementos de camélidos correspondientes a la MECiR

\subsection{Morfología cuantitativa de los camélidos}

\subsubsection{Análisis de componentes Principales (ACP)}

La aplicación del análisis de componentes principales (ACP) permite observar una variabilidad moderada en la muestra estudiada, ya que se necesitan considerar ocho componentes para resumir el 90,6\% de las variaciones. Los dos primeros componentes explican el $64,4 \%$ de la varianza total y expresan las principales varia- ciones morfológicas en las manifestaciones rupestres de camélidos (Fig.5).

El primer componente principal (CP1) resume el $50,7 \%$ de las variaciones correspondiente a los cambios en la longitud del cuerpo, en la postura y ancho de miembros, en las alturas a la grupa y a la cruz, postura del cuello; y morfología y disposición de la cola y oreja. De este modo, en los puntajes positivos se observan manifestaciones de camélidos con el cuerpo corto $\mathrm{y}$ ancho, miembros anteriores y posteriores anchos y dispuestos de modo recto hacia abajo, con alturas altas a la grupa, a la cruz y subesternal, y con la cola corta, ancha y dispuesta recta hacia arriba. Presentan el cuello recto hacia arriba, la cabeza de mayor tamaño en general (longitud y ancho de la cabeza y longitud de la cara); y con orejas anchas, cortas y dispuestas de modo recto también hacia arriba. Si observamos la distribución de las observaciones, se evidencia que estos caracteres definen una morfología vinculada a la MERH.

Por su parte, hacia los puntajes negativos se observan los elementos de cuerpo muy largo y angosto, miembros angostos y cortos (los posteriores rectos hacia abajo y los anteriores rectos hacia adelante en postura dinámica); con las alturas a la grupa, a la cruz y subesternal muy baja, y con la cola angosta, larga y flectada hacia abajo. Cuentan con el cuello recto inclinado hacia adelante, la cabeza de tamaño más reducido en general, y las orejas angostas, largas y flectadas hacia adelante. Las observaciones que presentan estos caracteres corresponden a la distribución de la MECiR sobre el CP1.

El segundo componente (CP2) resume $13,7 \%$ de las variaciones que corresponden principalmente a los cambios en la longitud, ancho y disposición del cuello, ancho del cuerpo, tamaño de la cola y tamaño general de la cabeza. De esta manera, en los puntajes positivos se registran los elementos con cuello muy largo, angosto e inclinado hacia adelante (más notable que en los puntajes negativos del primer componente), con el cuerpo más angosto, la cola flectada hacia abajo, pero de un tamaño reducido a prácticamente ausente, mientras que la cabeza es de tamaño reducido en todas las dimensiones que la conforman. Hacia los puntajes negativos se observan los camélidos con un cuello muy corto, ancho y recto hacia arriba, con el cuerpo ancho, la cola muy ancha, larga y recta hacia atrás, mientras que la cabeza presenta un gran tamaño en sus diferentes dimensiones, destacándose su considerable ancho. 


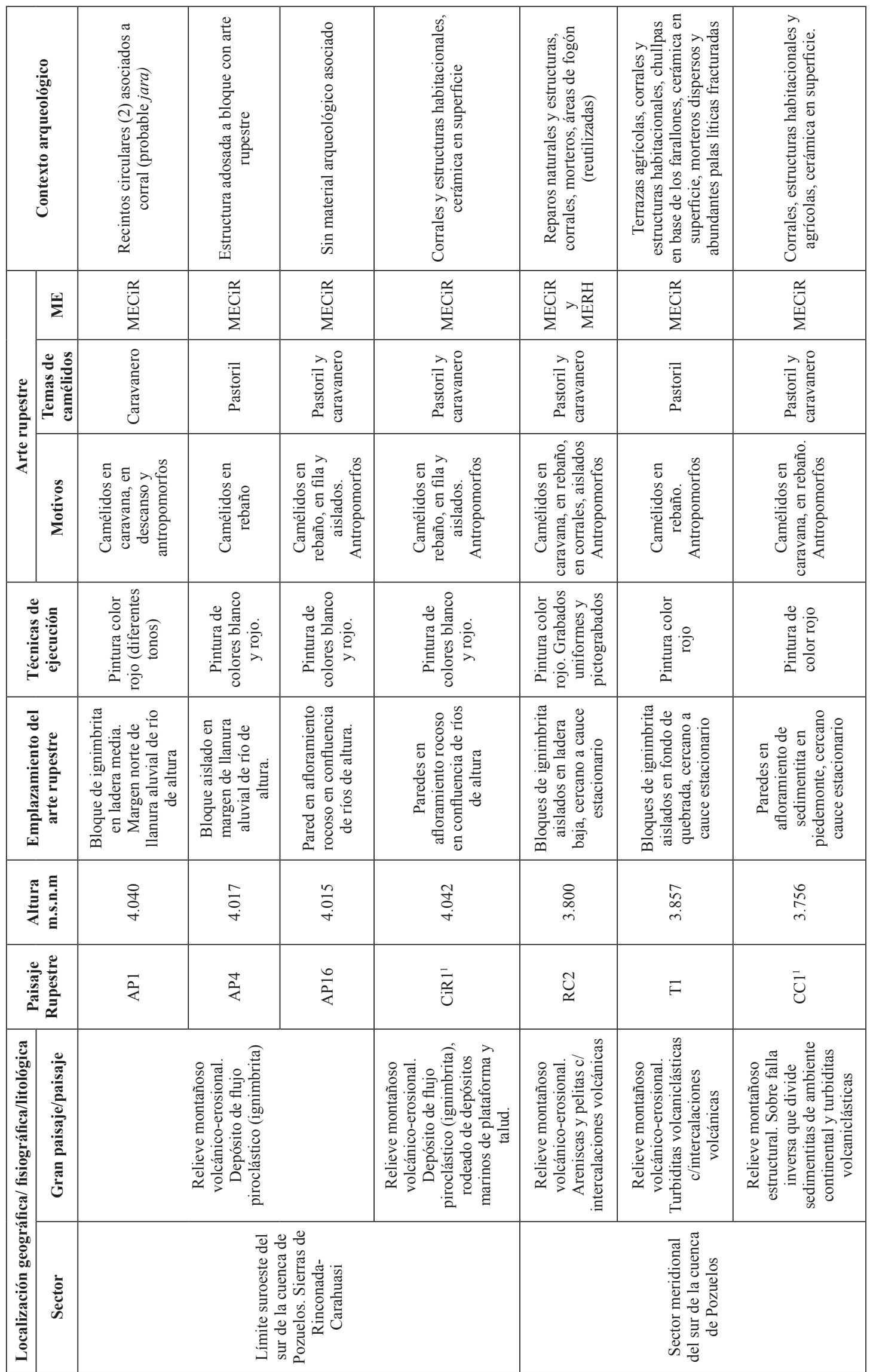




\begin{tabular}{|c|c|c|c|c|c|}
\hline & & 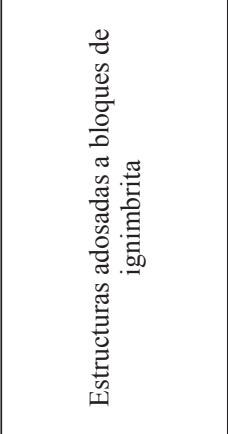 & 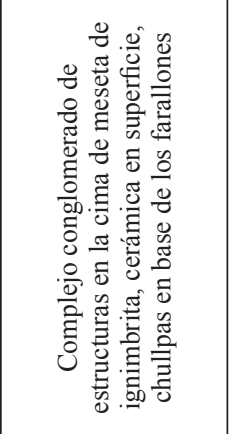 & 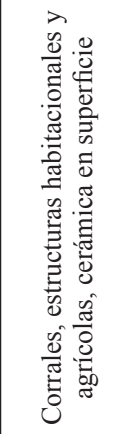 & \\
\hline \multirow{3}{*}{ 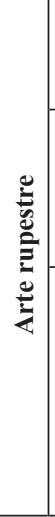 } & $\sum$ & $\begin{array}{l}\frac{\sqrt[T]{2}}{2} \\
\underset{1}{\Sigma}\end{array}$ & 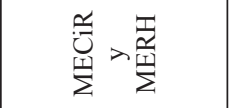 & 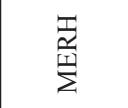 & \\
\hline & 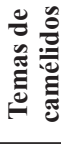 & 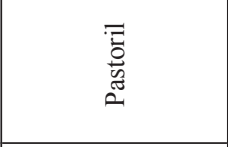 & 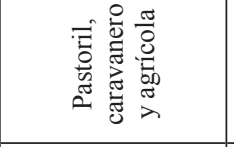 & 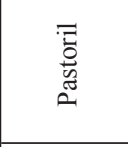 & \\
\hline & $\stackrel{\varrho}{E}$ & 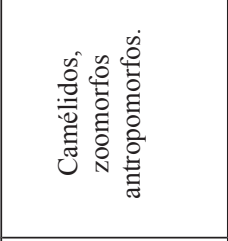 & 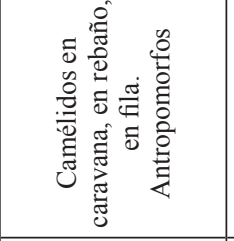 & 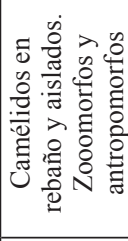 & \\
\hline \multicolumn{2}{|c|}{ 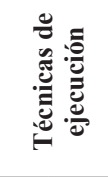 } & 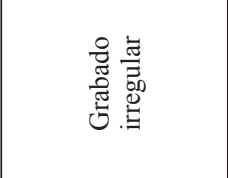 & 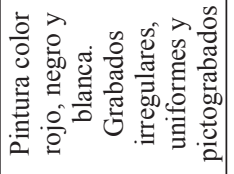 & 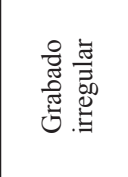 & 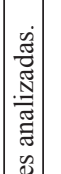 \\
\hline \multicolumn{2}{|c|}{ 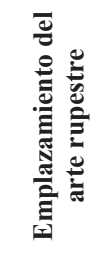 } & 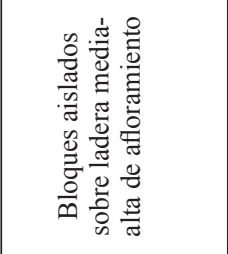 & 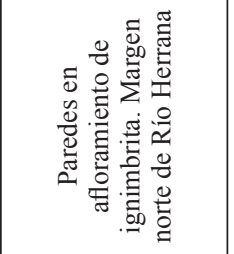 & 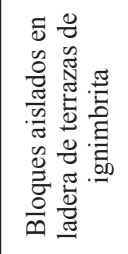 & 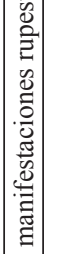 \\
\hline \multicolumn{2}{|c|}{ 总 } & $\begin{array}{l}\stackrel{\infty}{\curvearrowright} \\
\dot{r}\end{array}$ & $\begin{array}{l}\stackrel{D}{\circ} \\
\dot{m}\end{array}$ & $\begin{array}{l}\hat{s} \\
\infty \\
\dot{n}\end{array}$ & 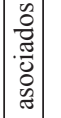 \\
\hline \multicolumn{2}{|c|}{ 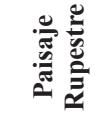 } & ชิ & $\stackrel{\mathfrak{\Xi}}{\exists}$ & $\stackrel{\circ}{\stackrel{1}{a}}$ & 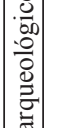 \\
\hline \multirow{2}{*}{ 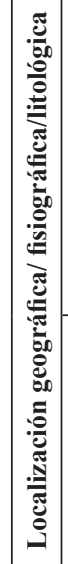 } & 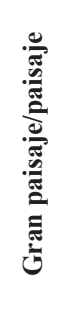 & 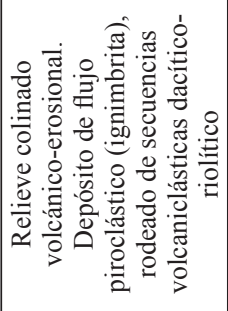 & \multicolumn{2}{|c|}{ 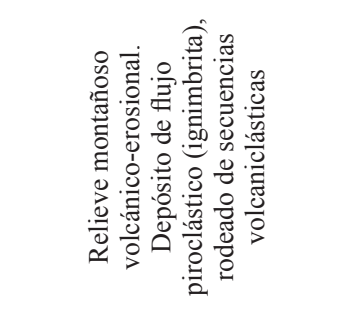 } & 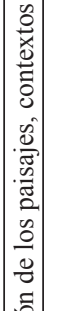 \\
\hline & क्ष̈ & & 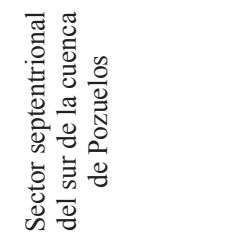 & & 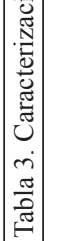 \\
\hline
\end{tabular}



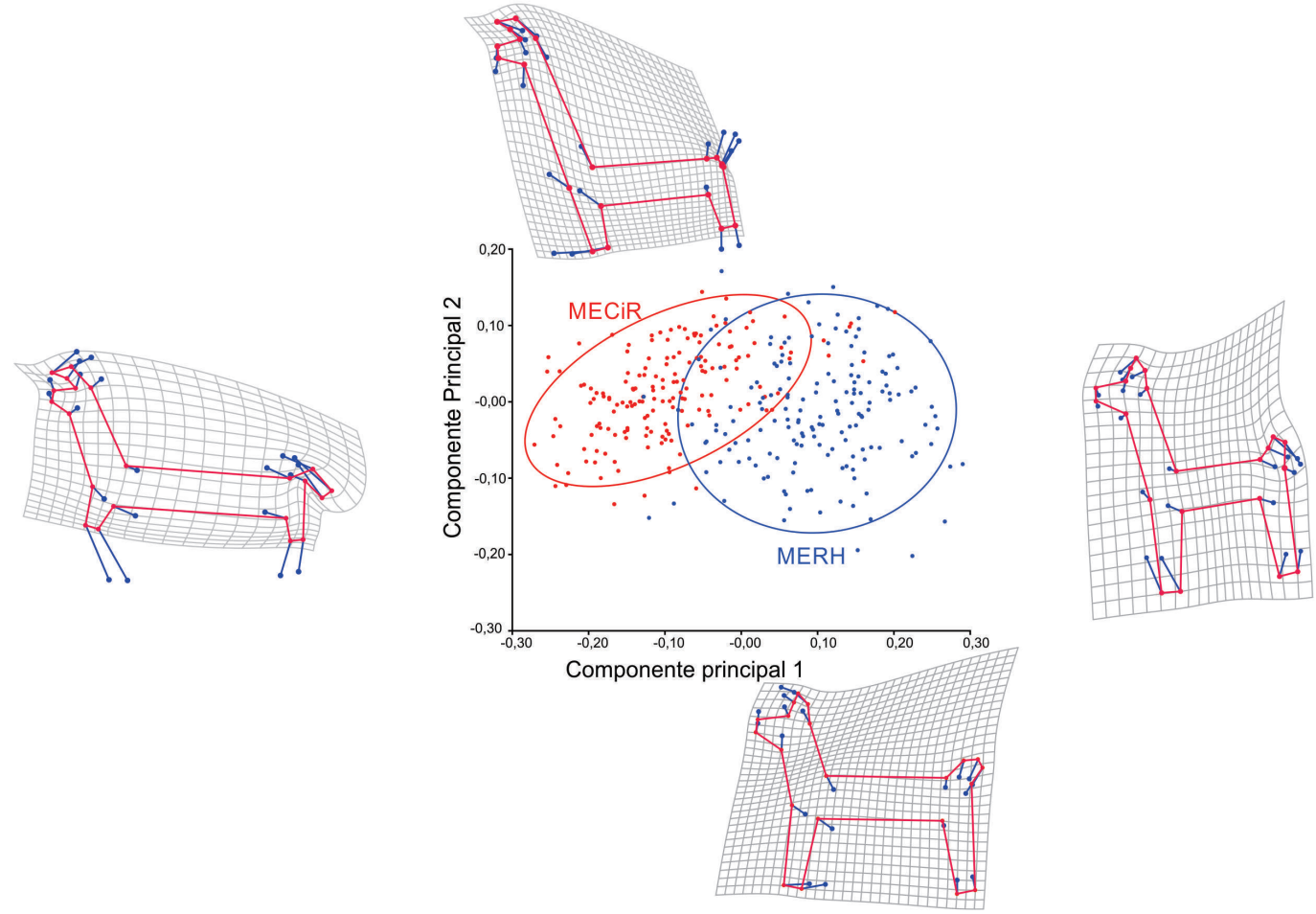

Figura 5. Análisis de componentes principales (PC1 y PC2) sobre las coordenadas de forma de Procrustes, con elipses de confianza (0.9) y con contornos y cuadrículas de deformación.

En la figura 5, se presentan las grillas de deformación donde se observan las referidas variaciones morfológicas en la configuración de las manifestaciones de camélidos correspondientes a los puntajes más extremos del primer componente $(-0.3$ y 0.3$)$, y los puntajes más extremos del segundo componente (-0.2 y 0.2$)$, mientras que en los valores intermedios se observan diferentes grados de dichas variaciones morfológicas.

Como ya mencionamos, cada uno de los puntos en el espacio entre los ejes representa a cada una de las manifestaciones de camélidos. Al presentar dichos puntos vinculados a cada una de las modalidades estilísticas definidas (MERH y MECiR), se observa en general una mayor dispersión de los datos en la MERH en relación a los de la MECiR. Sin embargo, mientras en la MERH esta dispersión ocurre de manera similar en relación a ambos ejes, en la MECiR la dispersión mayor se da sobre el primer componente. Esta situación implica una mayor diversidad morfológica de los camélidos de la MERH en relación a los de la MECiR y de este modo, se pueden vincular estas ten- dencias a las propiedades morfológicas que caracterizarían a cada modalidad estilística como puede observarse en las cuadrículas de deformación de la figura 5.

\subsubsection{ANOVA de Procrustes}

Las variaciones observadas a través del ACP pudieron ser cuantificadas a través del ANOVA de Procrustes, que indica una diferencia significativa en relación al tamaño del centroide entre modalidades estilísticas $(\mathrm{F}=30.30$, gl 1, $\mathrm{p}<0.0001)$, y en relación a los paisajes rupestres $(\mathrm{F}=14.66$, $\mathrm{gl} 9, \mathrm{p}<0.0001)$. Este mismo análisis aplicado en relación a la forma propiamente dicha (shape) también arrojó diferencias significativas tanto para las modalidades estilísticas $(\mathrm{F}=158.61, \mathrm{gl}$ $38, \mathrm{p}<0.0001$; Pillai $=0.88, \mathrm{p}<0.0001$ ), como para los paisajes rupestres $(\mathrm{F}=4.90, \mathrm{gl} 342, \mathrm{p}<0.0001$; Pillai ${ }^{9}=2.53, p<0.0001$ ). La forma consenso y las variaciones morfológicas observadas en la configuración de las manifestaciones rupestres de camélidos para ambas modalidades estilísticas se puede observar en la figura 6 a través de los contornos promedio correspondientes a cada una. 

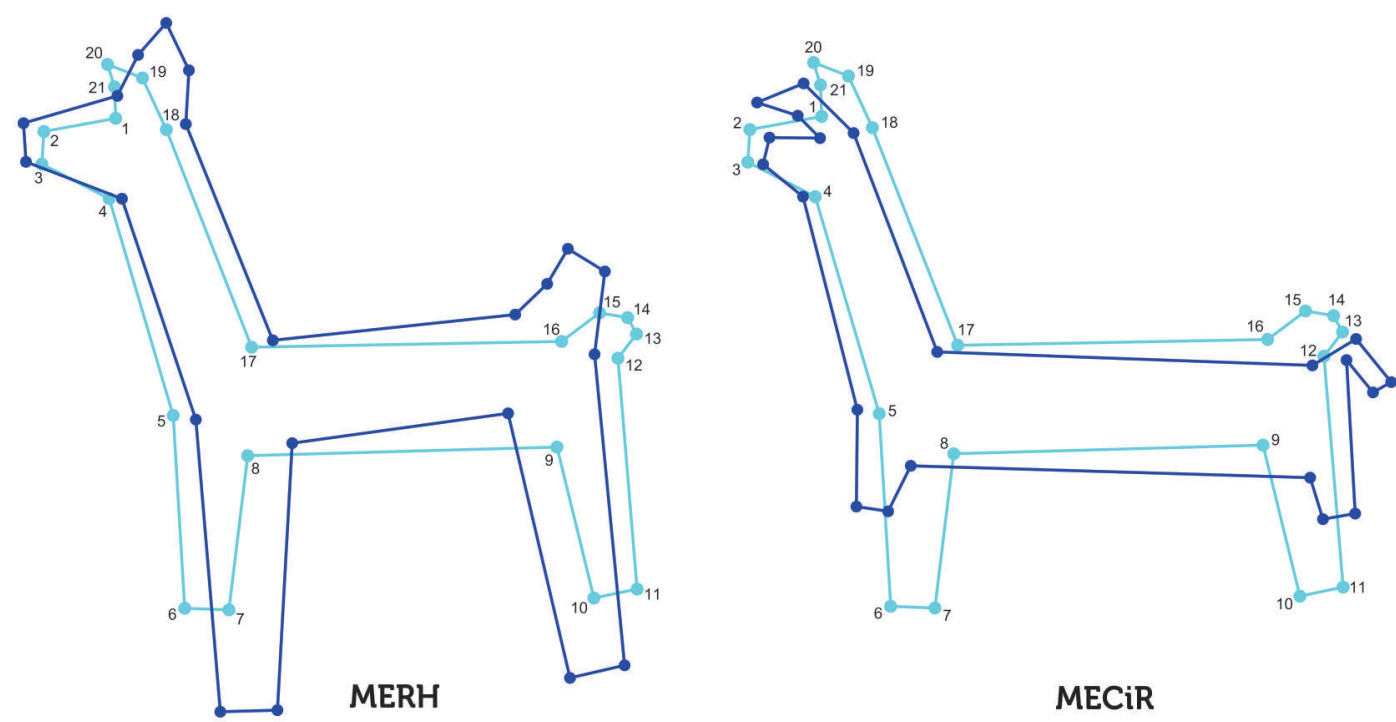

Figura 6. Contornos de las formas consenso (Procrustes) para cada una de las modalidades estilística MERH y MECiR.

\subsubsection{Regresión Multivariada}

Para analizar si existe relación entre las diferentes morfologías de camélidos y los diferentes tamaños de los mismos (alometría) se realizó un análisis de Regresión Multivariada (Zelditch et al. 2014; Klingenberg 2009; Johnson 2000). Para ello se consideró como variable independiente el tamaño (estimado a través de log centroide size $e^{10}$ ) y como variables dependientes a las coordenadas de forma alineadas (Procrustes coordinates). La hipótesis nula sostiene que las variables tamaño y forma propiamente dicha son independientes y por tanto no habría covarianza entre ellas. El análisis de regresión arrojó resultados significativos $(p<0.0001$, considerando 10.000 permutaciones contra la hipótesis nula), evidenciando la dependencia de la forma en relación al tamaño, pero con una baja incidencia, ya que solo el $2.73 \%$ de las formas son predichas por el tamaño (suma total de cuadrados=
10,996; suma de cuadrados predicha $=0,299$ ). Esto implica que el $97.28 \%$ de las variaciones morfológicas no son explicadas por el tamaño y conforman los residuos de la regresión (suma de cuadrados residual $=10,696$ ). Estos residuos, que ya no cuentan con el efecto del tamaño, serán utilizados para los análisis de modularidad e integración morfológica a continuación.

Resulta interesante destacar, que al realizar este tipo de análisis en relación a cada una de las modalidades estilísticas se observa que si bien en ambas la covariavión entre la forma propiamente dicha y el tamaño es significativa ( $\mathrm{P}<0.0001$ ), en la MECiR la influencia de la alometría (efecto del tamaño sobre la forma) es mayor $(6.53 \%)$ que en relación a la MERH $(4.57 \%)$ (Tabla 4$)$. De este modo se observa que, si bien la alometría es una de las causas en los cambios morfológicos de ambas modalidades, esta situación se registra en mayor proporción para la MECiR (Fig. 7).

Tabla 4. Suma de cuadrados total, suma de cuadrados predicho, suma de cuadrados residual, porcentaje de la forma predicho por el tamaño, cantidad de permutaciones contra la hipótesis nula y P-valor para evaluar el efecto de la alometría en cada modalidad estilística.

\begin{tabular}{|l|c|c|c|c|c|c|}
\hline $\begin{array}{c}\text { Modalidad } \\
\text { Estilística }\end{array}$ & Total SC & SC predicho & SC residual & $\%$ predicho & N permutaciones & $P$-valor \\
\hline MECiR & 3,25580518 & 0,21282692 & 3,04297827 & $6,5368 \%$ & 10.000 & $<0.0001$ \\
\hline MERH & 4,51104832 & 0,20645422 & 4,30459410 & $4,5766 \%$ & 10.000 & $<0.0001$ \\
\hline
\end{tabular}




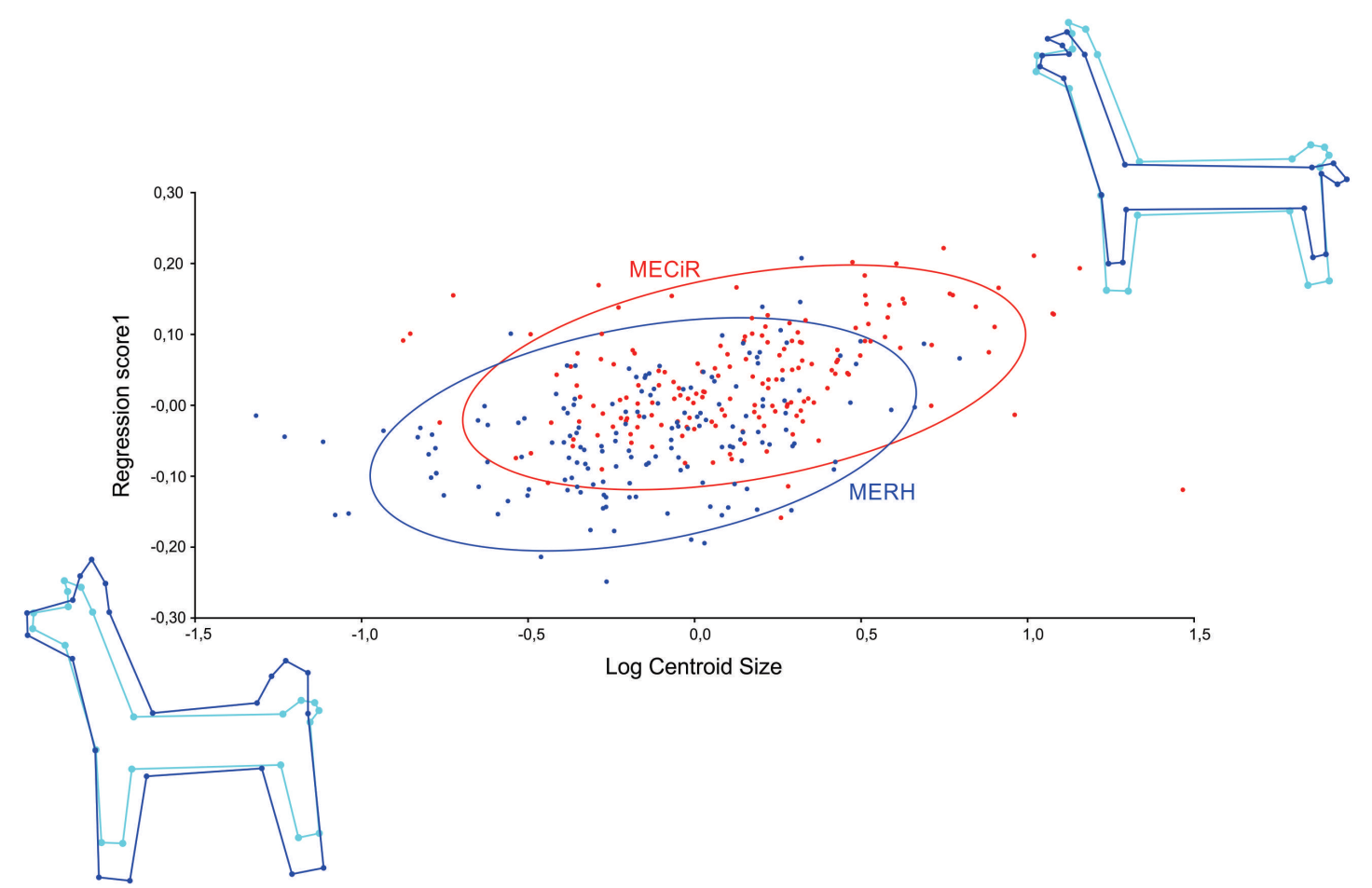

Figura 7. Regresión tamaño-forma de las manifestaciones de camélidos en el sur de Pozuelos. Los puntos de colores identifican las observaciones correspondientes a los diez paisajes rupestres, mientras que las elipses de confianza (0.9) corresponden a las modalidades estilísticas (MERH y MECiR)

\subsubsection{Modularidad}

En primera instancia, para probar la hipótesis de la existencia de modularidad en la manufactura de los camélidos de ambas modalidades estilísticas, se utilizaron las matrices de covariación para examinar dos subgrupos de landmarks definidos a priori, probando particiones contiguas y no contiguas evaluadas mediante 10.000 permutaciones. Es importante destacar que para estas pruebas de modularidad se utilizaron los residuos del análisis de Regresión Multivariada. Esta decisión se tomó con el fin de eliminar el efecto de la alometría, y de este modo, las matrices de covariación de los residuos de la regresión permiten abordar las variaciones de la forma propiamente dicha (shape) después de extraer la influencia del tamaño (Klingenberg 2009).

Para ello, y guiándonos con los resultados obtenidos en el ACP, los landmarks fueron subdivididos en aquellos que describen los caracteres de longitud del cuerpo, altura a la grupa y a la cruz como subconjunto A (landmarks: $5,6,7,8,9,10,11,16,17)$ y por otro lado, los que describen longitud del cuello, y morfología de cabeza, oreja y cola como subconjunto
B (landmarks: 1, 2, 3, 4, 12, 13, 14, 15, 18, 19 , 20,21) (Fig. 8a). Dicha prueba arrojó un coeficiente $\mathrm{RV}^{11}=0.731$, mientras que la proporción de particiones con un RV menor o igual a la hipótesis definida a priori es de 0.0095 , siendo la partición con el mínimo $\mathrm{RV}=0.583$.

La existencia de modularidad se entiende como la división de unidades, en este caso subconjuntos de puntos que definen ciertos rasgos de los camélidos, que cuentan con fuertes interacciones entre sí, es decir, altamente integradas en su interior, pero que al mismo tiempo son relativamente independientes de otras unidades (módulos o subconjuntos de puntos) (Klingenberg 2009). En nuestro caso, la hipótesis de modularidad se mantiene porque el coeficiente de RV para la partición hipotética tiene un valor que se ubica cerca del extremo inferior de la distribución de los coeficientes de RV para todas las particiones probadas, con solo 95 particiones de las 10.000 probadas que presentan un RV menor al de la partición hipotética, sin embargo, el grado de modularidad es bajo.

Al analizar la independencia relativa de estos hipotéticos módulos en relación a cada modalidad estilística por separado, encontramos un dato 
interesante, si bien los valores del coeficiente de RV para ambas modalidades se ubican cerca del extremo inferior de la distribución de dichos coeficientes (flechas en histograma de Fig. 8b y c), que permiten sostener la existencia de modularidad (en un grado bajo en general), la dependencia relativa entre los módulos definidos es bastante mayor en la MECiR $(\mathrm{RV}=0.785$, con 2340 particiones con RV menor a la hipótesis) y menor en la MERH (RV=0.562, 105 particiones con RV menor a la hipótesis). Esto quiere decir que en la MECiR el grado de modularidad en la configuración del camélido es menor que en relación al grado de modularidad de la MERH (Fig. 8c). Estos resultados son consistentes con la hipótesis de que los subconjuntos A y B pueden ser considerados como módulos distintos con una relativa independencia que varía en relación a las modalidades estilísticas, situación que también tiene relación con la integración morfológica observada en la figura del camélido abordada a continuación mediante el Análisis de Mínimos Cuadrados Parciales (Klingenberg 2009).

Para evaluar el efecto del tamaño sobre la hipótesis de modularidad, consideramos las matrices de covarianza sin la corrección de la alometría (Tabla 5). Esto permite observar para ambas modalidades estilísticas un aumento en el valor de los coeficientes de RV, una disminución en las particiones alternativas con RV menor al de la hipótesis. Esta situación implica que al incluir la influencia del tamaño se disminuye el grado de modularidad (aumenta la covariación entre módulos) y por tanto aumenta el grado de dependencia entre los subconjuntos A y B.
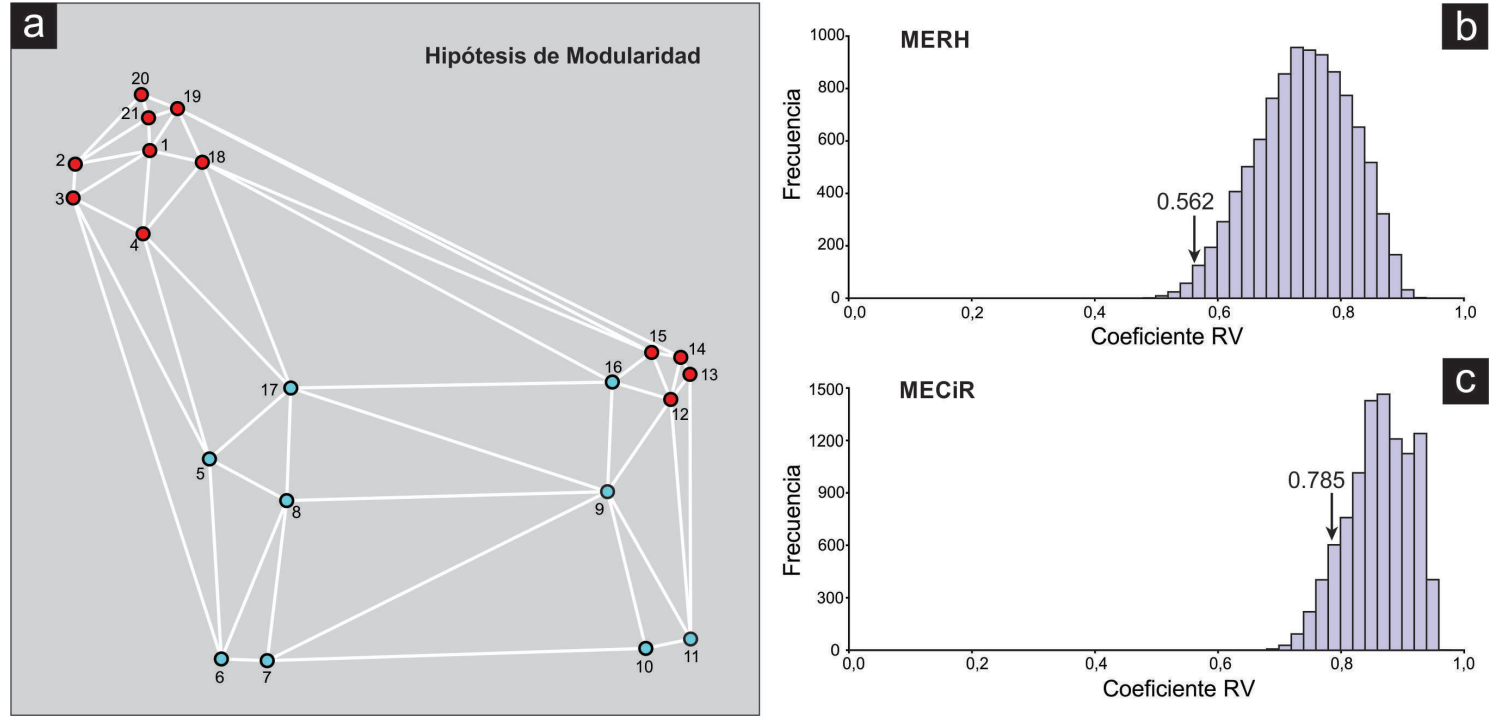

Figura 8. a. Esquema de hipótesis de modularidad, definición de subconjuntos A (celeste) y B (rojo).

b y c. Histogramas de la distribución de los coeficientes RV generados por 10.000 permutaciones sin efecto del tamaño (free size). La flecha negra muestra la ubicación de nuestra hipótesis en la curva de distribución: (b) para la MERH un RV de 0.562, (c) para la MECiR un RV de 0.785.

Tabla 5. Coeficientes RV, número de particiones con RV menor o igual a la hipótesis a priori y partición con RV mínimo, 10.000 particiones contiguas y no contiguas consideradas en cada caso con y sin efecto de alometría para la MERH y la MECiR.

\begin{tabular}{|lcccc|}
\hline $\begin{array}{c}\text { Modalidad } \\
\text { Estilística }\end{array}$ & Influencia de la alometría & $\begin{array}{c}\text { Coeficiente RV de } \\
\text { hipótesis }\end{array}$ & $\begin{array}{c}\text { N particiones con RV } \\
\text { menor o igual que } \\
\text { hipótesis }\end{array}$ & $\begin{array}{c}\text { Partición con RV } \\
\text { mínimo }\end{array}$ \\
\hline \multirow{2}{*}{ MECiR } & Con efecto de tamaño & 0,797 & 1112 & 0,656 \\
\cline { 2 - 5 } & Sin efecto de tamaño & 0,785 & 918 & 0,680 \\
\hline \multirow{2}{*}{ MERH } & Con efecto de tamaño & 0,581 & 166 & 0,490 \\
\cline { 2 - 5 } & Sin efecto de tamaño & 0,562 & 105 & 0,489 \\
\hline
\end{tabular}




\subsubsection{Mínimos Cuadrados Parciales}

El análisis de Mínimos Cuadrados Parciales (PLS en inglés) ${ }^{12}$ (Bookstein et al. 2003; Klingenberg 2009) permitió estimar la fuerza de covariación de ciertos caracteres y el grado de vinculación entre ellos para abordar la integración morfológica como cambios específicos en dichos caracteres que puedan ocurrir de manera coordinada. Se probó la modularidad entre las regiones anatómicas referidas, a partir de la definición de dos bloques con los subconjuntos A y B empleados en la hipótesis de modularidad, que confirmó el coeficiente
$\mathrm{RV}=0.731, \mathrm{p}<0.0001$, con rondas de 10.000 permutaciones. El total de las covariaciones entre los bloques 1 y 2 se expresa en 18 coeficientes PLS, mientras que el mayor porcentaje de esta covariación se resume en el coeficiente PLS1 (91.76\%), con una correlación de 0.89 , $\mathrm{p}<0.0001$, el cual se puede visualizar en la figura 9 donde se observan también los cambios de forma de los camélidos en relación a los ejes mostrando las características de la forma que representan la mayor covariación entre las partes probadas, en lugar de la variación general en toda la estructura analizada (como presentamos en el ACP).

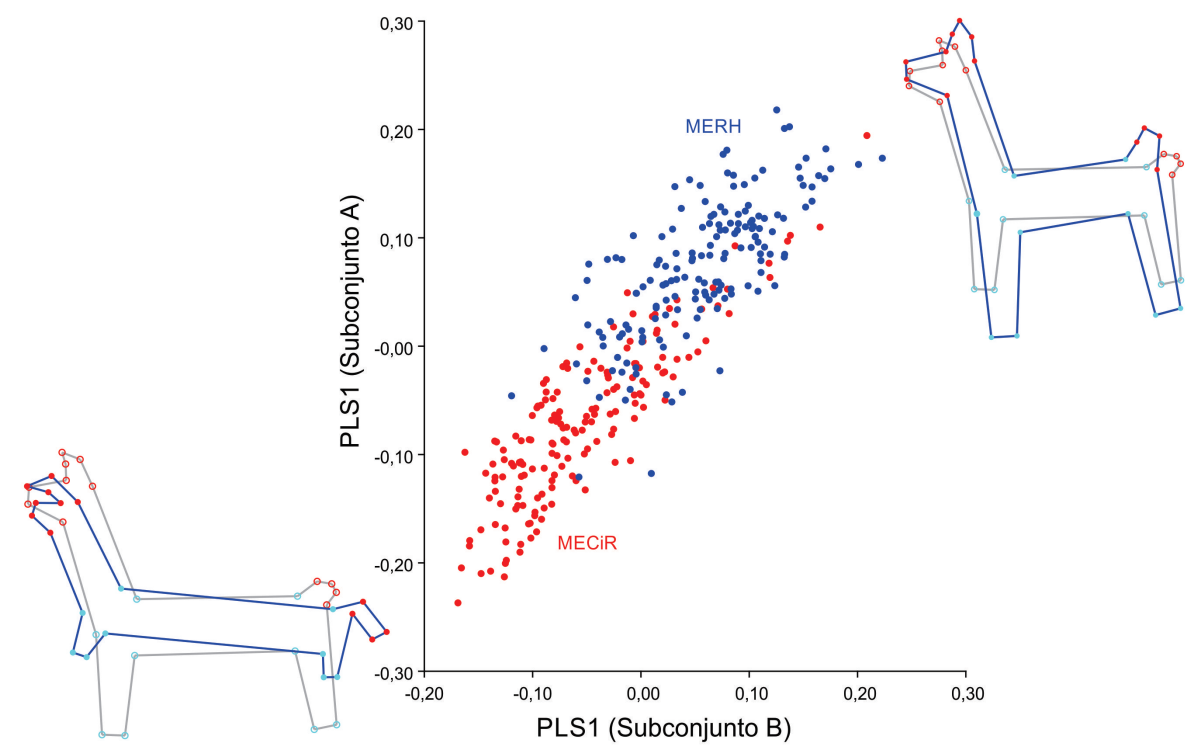

Figura 9. Resultado del PLS y contornos de camélidos con los cambios de forma en relación a los ejes mostrando las características de la forma que representan la mayor covariación. En rojo elementos de la MECiR, en azul los de la MERH.

Tabla 6. Correlación por pares de puntajes PLS 1 y 2 entre bloques (subconjuntos de hitos A y B) en relación a las modalidades estilísticas MERH y MECiR con y sin efecto del tamaño, todos ellos con 10.000 permutaciones.

\begin{tabular}{|c|c|c|c|c|c|}
\hline \multicolumn{6}{|l|}{ MERH } \\
\hline & Coeficiente RV & & Covariación total (\%) & Correlación & $P$-valor \\
\hline \multirow{2}{*}{$\begin{array}{l}\text { Con efecto del } \\
\text { tamaño }\end{array}$} & \multirow{2}{*}{$0.581 ; \mathrm{P}<0.0001$} & PLS1 & 61,088 & 0,88224 & 0,0268 \\
\hline & & PLS2 & 27,384 & 0,88503 & 0,0001 \\
\hline \multirow{2}{*}{$\begin{array}{l}\text { Sin efecto del } \\
\text { tamaño }\end{array}$} & \multirow{2}{*}{$0.562 ; \mathrm{P}<0.0001$} & PLS1 & 56,107 & 0,87866 & 0,0225 \\
\hline & & PLS2 & 31,554 & 0,87723 & 0,0001 \\
\hline \multicolumn{6}{|l|}{ MECiR } \\
\hline & Coeficiente RV & & Covariación total (\%) & Correlación & $P$-valor \\
\hline \multirow{2}{*}{$\begin{array}{l}\text { Con efecto del } \\
\text { tamaño }\end{array}$} & \multirow{2}{*}{$0.797 ; \mathrm{P}<0.0001$} & PLS1 & 88,950 & 0,93182 & $<0.0001$ \\
\hline & & PLS2 & 8,359 & 0,90503 & $<0.0001$ \\
\hline \multirow{2}{*}{$\begin{array}{l}\text { Sin efecto del ta- } \\
\text { maño }\end{array}$} & \multirow{2}{*}{$0.785 ; \mathrm{P}<0.0001$} & PLS1 & 88,580 & 0,92954 & $<0.0001$ \\
\hline & & PLS2 & 8,353 & 0,88860 & $<0.0001$ \\
\hline
\end{tabular}


Estos resultados indican una alta y significativa correlación entre los dos subconjuntos de variables definidas, lo que implica un alto grado de integración morfológica en las manifestaciones de camélidos, es decir una configuración estandarizada o coordinada que, por ejemplo, al conocer una determinada morfología del cuerpo y los miembros anteriores y posteriores (subconjunto A) vinculada a una modalidad, permitiría inferir aproximadamente la morfología del cuello, cabeza, oreja y cola relacionada a ella (subconjunto B). Asimismo, esta coordinación o integración morfológica se estructura modularmente como vimos anteriormente, permitiendo cierta independencia de los dos subconjuntos definidos.

Del mismo modo que se observó en la prueba de hipótesis de la Modularidad, en el análisis de $P L S$ se identificaron también ciertas diferencias en relación a las modalidades estilísticas. En la tabla 6 se presentan los valores obtenidos para la MERH y la MECiR sobre la covariación total, correlación y el p-valor de los coeficientes de PLS 1 y 2. Allí se puede observar que los PLS1 y 2 de la MERH alcanzan el $88.472 \%$ de la covariación total, mientras en la MECiR alcanza el 97.309\% (teniendo el PLS1 el 88.95\%). Si bien en ambas modalidades los coeficientes PLS presentan una alta correlación, se destaca que para la MECiR no solo es mayor al 0.9 , sino que dicha correlación es significativa $(P$-valor $<0.0001)$ no siendo del mismo modo para la MERH. Estos resultados indican que los subconjuntos analizados presentan mayor independencia en la MERH (vinculado también al mayor grado de modula- ridad) que en la MECiR y, por tanto, esta última cuenta con una mayor integración morfológica en la composición de la figura del camélido. En ambas modalidades estilísticas podemos ver que el efecto de la alometría aumenta el coeficiente $\mathrm{RV}$, la covariación resumida en los PLS 1 y 2 , la correlación entre bloques y por tanto la integración morfológica de la figura del camélido.

\subsubsection{Análisis de Variantes Canónicas}

La aplicación del análisis de variantes canónicas (AVC) permite la reducción de la dimensionalidad, la comparación entre medias grupales y evaluar dónde ocurren las diferencias más importantes entre las medias de los grupos definidos (Johnson 2000), por una parte, en relación a las modalidades estilísticas MERH y MECiR, y por otra, en relación a los diez paisajes rupestres considerados. Al aplicar este análisis en relación a las modalidades estilísticas, observamos que el 100\% de la varianza de la muestra se expresa en la variante canónica 1 , mientras que las diferencias estadísticas sobre similitud o diferencia entre los grupos fueron evaluadas por test no paramétrico como la permutación, que arrojaron diferencias significativas entre modalidades estilísticas con una distancia de Mahalanobis de 4.497 ( $\mathrm{p}<0.0001$, con 10.000 permutaciones) y una distancia de Procrustes de $0.201(p<0.0001$, con 10.000 permutaciones). En la figura 10 puede observarse la forma de camélido más frecuente para cada modalidad estilística.

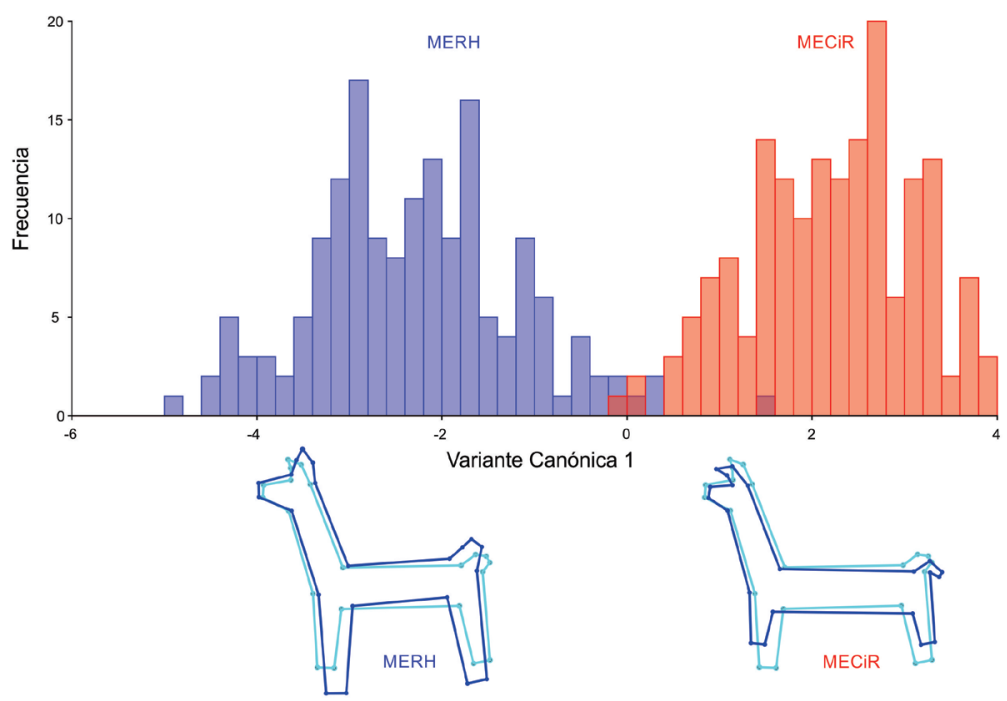

Figura 10. Análisis de AVC para las modalidades estilísticas. Discriminación más efectiva en la Variante Canónica 1 que diferencia notablemente a la MERH de la MECiR. Se presentan además los contornos de camélidos más frecuentes para cada modalidad. 
Este mismo análisis aplicado en relación a los diez paisajes rupestres estudiados permite observar que las variantes canónicas 1 y 2 explican el $70.99 \%$ de las variaciones entre grupos, $(58.12 \%$ y $12.87 \%$ respectivamente). En la figura 11 se observa la distribución de las morfologías de los camélidos en relación a los diferentes paisajes rupestres y en las tablas 8 y 9 se observan las diferencias estadísticas entre estos grupos a través de las distancias de Mahalanobis y de Procrustes considerando un nivel de significación de 0.0001, arrojando diferencias significativas (en base a 10.000 permutaciones) entre algunos de los paisajes rupestres. Esto plantea el rechazo de la hipótesis nula acerca de la no existencia de diferencia entre cada par de grupos y, por tanto, permite comprobar la existencia de diferencias morfológicas en los camélidos de los paisajes rupestres considerados. Si bien algunas de estas diferencias se explican por su pertenencia a la MERH o la MECiR, se observan diferencias entre los paisajes rupestres que no solo estarían explicadas por dichas diferencias entre las modalidades.

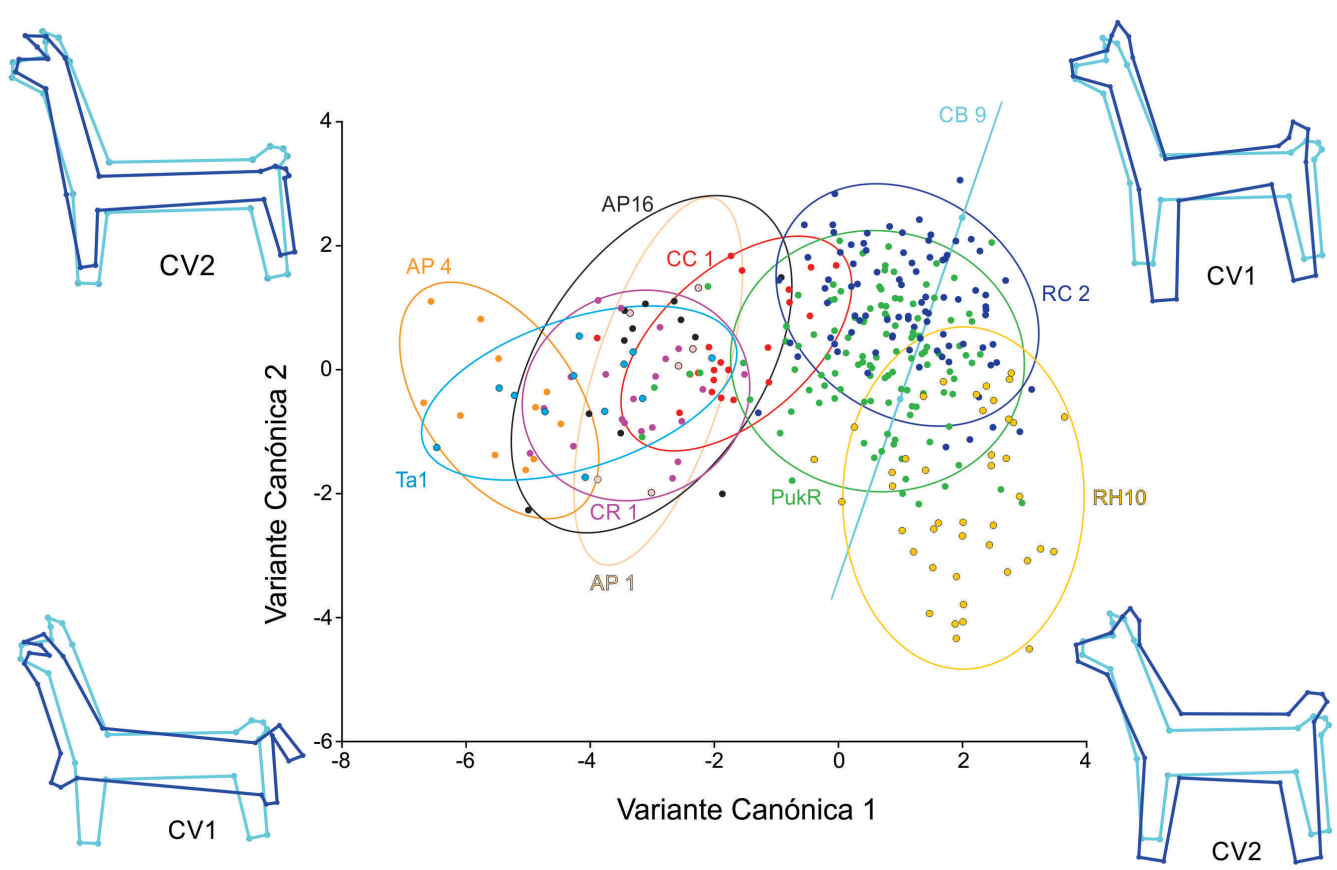

Figura 11. Análisis de Variantes Canónicas. Distribución de las morfologías de los camélidos en relación a los paisajes rupestres.

Tabla 7. Reclasificación de las observaciones en cada modalidad estilística por medio de la función discriminante

\begin{tabular}{|l|l|l|l|}
\hline Modalidad Estilística & \multicolumn{1}{|c|}{ MECiR (n) \% } & \multicolumn{1}{|c|}{ MERH (n) \% } & \multicolumn{1}{c|}{ Total (n) \% } \\
\hline MECiR & $(165) 98.21 \%$ & $(3) 1.78 \%$ & $(168) 100 \%$ \\
\hline MERH & $(8) 95.06 \%$ & $(154) 4.93 \%$ & $(162) 100 \%$ \\
\hline
\end{tabular}

\subsubsection{Análisis Discriminante}

Para abordar las diferencias del AVC, se aplicó el procedimiento del Análisis Discriminante para comprobar la correcta asignación a la modalidad estilística de cada camélido mediante la reclasificación de las observaciones según la mayor probabilidad de pertenecer a una $\mathrm{u}$ otra modalidad estilística. Los resultados con- firman las distancias de Mahalanobis y de Procrustes mencionadas previamente, y también permite observar una baja reclasificación de camélidos en una modalidad distinta a su pertenencia original, es decir que en ambos casos se alcanza un valor de acierto considerable en la asignación de cada camélido a las modalidades estilísticas diferenciadas previamente (Tabla 7). 
Al aplicar dicho análisis en los paisajes rupestres de a pares y en relación a la modalidad estilística a la que han sido clasificados a prio$r i$, se obtuvieron las distancias de Procrustes, de Mahalanobis y sus $P$-valor para cada par, con 10.000 permutaciones para cada caso.

De estas matrices de distancias se desprenden varias consideraciones, en primera instancia que entre los paisajes rupestres que pertenecen a distintas modalidades estilísticas se confirma en todos los casos diferencias significativas $(p<0.0001)$ entre las morfologías de las manifestaciones de camélidos tanto para la distancia de Procrustes como para la distancia de Mahalanobis (valores sombreados en gris en las Tablas 8 y 9). Esta situación confirma entonces que, las diferencias identificadas entre modalidades estilísticas a través de todos los análisis previos también se reflejan claramente entre los paisajes que presentan diferentes modalidades (Fig. 12a), incluso cuando dichas modalidades se encuentran compartiendo el mismo paisaje, como ocurre en el Pukara de Rinconada y en Río Candado 2, que aun así las diferencias entre ellas son significativas. a

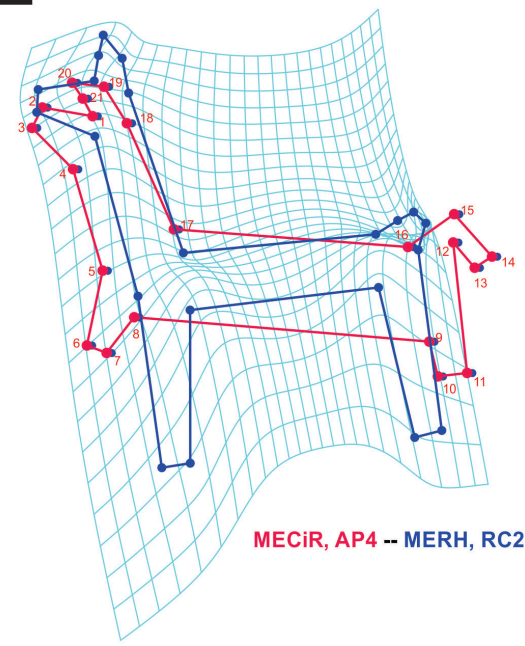

b

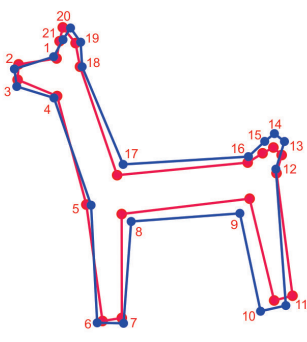

MERH, RC2 -- MERH, RH10

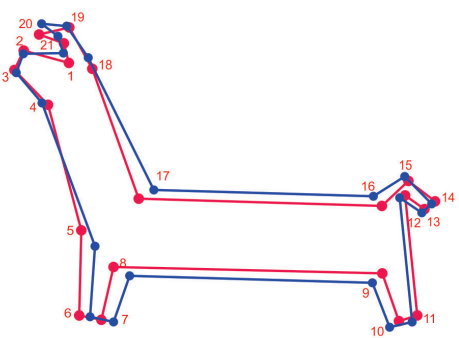

MECiR, AP16 -- MECiR, CiR1

Figura 12. Diferencias morfológicas entre las formas promedio de cada paisaje rupestre en relación a la modalidad estilística. a. Máxima distancia de Procrustes entre modalidades estilísticas observada entre los paisajes rupestres de AP4 (MECiR) y RC2 (MERH). b. Menor distancia de Procrustes intra MERH observada entre los paisajes rupestres de RC2 y RH10. c. Menor distancia de Procrustes intra MECiR observada entre los paisajes rupestres de AP16 y CiR1.

En segunda instancia, este análisis nos permitió identificar también diferencias significativas entre paisajes rupestres dentro de una misma modalidad estilística, esto resulta interesante porque más allá de las claras diferencias entre MERH y MECiR, nos permite visualizar una variabilidad interna dentro de cada modalidad (Fig. 12b y c). Estas distancias entre paisajes rupestres se pueden visualizar claramente en la figura 11, donde cada una de las elipses de confianza ( 0.9 de probabilidad) expone las relaciones entre los ejes de las variantes canónicas que resumen dichas diferencias y los ubica sobre la VC1. Desde el cero hacia los valores negativos se encuentran los paisajes vinculados a la MECiR, mientras que hacia los puntajes positivos se ubican los vinculados a la MERH. Las posiciones y distancias relativas entre ambos ejes expresan de manera gráfica los valores presentados de las distancias entre paisajes. 
Tabla 8. Distancias de Mahalanobis entre pares paisajes rupestres en relación a las modalidades estilísticas (hemimatriz inferior) y P-values de dichas distancias (10000 permutaciones) (hemimatriz superior). Sin efecto del tamaño (free size), considerando solo la forma propiamente dicha. En negrita las distancias significativas.

\begin{tabular}{lccccccccccc}
\hline $\begin{array}{l}\text { Paisajes } \\
\text { rupestres }\end{array}$ & $\begin{array}{c}\text { AP1- } \\
\text { MECiR }\end{array}$ & $\begin{array}{c}\text { AP4- } \\
\text { MECiR }\end{array}$ & $\begin{array}{c}\text { AP16- } \\
\text { MECiR }\end{array}$ & $\begin{array}{c}\text { CC1- } \\
\text { MECiR }\end{array}$ & $\begin{array}{c}\text { CR1- } \\
\text { MECiR }\end{array}$ & $\begin{array}{c}\text { PuR- } \\
\text { MECiR }\end{array}$ & $\begin{array}{c}\text { PuR- } \\
\text { MERH }\end{array}$ & $\begin{array}{c}\text { RC2- } \\
\text { MECiR }\end{array}$ & $\begin{array}{c}\text { RC2- } \\
\text { MERH }\end{array}$ & $\begin{array}{c}\text { RH10- } \\
\text { MERH }\end{array}$ & $\begin{array}{c}\text { T1- } \\
\text { MECiR }\end{array}$ \\
\hline $\begin{array}{l}\text { AP1- } \\
\text { MECiR }\end{array}$ & & 0,7896 & 0,8440 & 0,9191 & 0,9042 & 0,0002 & $<.0001$ & 0,0251 & $<.0001$ & $<.0001$ & 0,8690 \\
$\begin{array}{l}\text { AP4- } \\
\text { MECiR }\end{array}$ & 5,8183 & & 0,6532 & 0,5857 & 0,6542 & $<.0001$ & $<.0001$ & $<.0001$ & $<.0001$ & $<.0001$ & 0,4953 \\
$\begin{array}{l}\text { AP16- } \\
\text { MECiR }\end{array}$ & 5,3739 & 9,1089 & & 0,8046 & 0,5276 & $<.0001$ & $<.0001$ & $<.0001$ & $<.0001$ & $<.0001$ & 0,8184 \\
$\begin{array}{l}\text { CC1- } \\
\text { MECiR }\end{array}$ & 6,3622 & 13,4892 & 8,4776 & & 0,2483 & 0,0003 & $<.0001$ & $<.0001$ & $<.0001$ & $<.0001$ & 0,6334 \\
$\begin{array}{l}\text { CR1- } \\
\text { MECiR }\end{array}$ & 6,6851 & 11,6567 & 15,4203 & 10,9429 & & $<.0001$ & $<.0001$ & $<.0001$ & $<.0001$ & $<.0001$ & 0,7822 \\
$\begin{array}{l}\text { PuR- } \\
\text { MECiR }\end{array}$ & 21,6068 & $\mathbf{1 3 , 7 4 2 7}$ & $\mathbf{1 2 , 4 0 4 0}$ & 5,2933 & $\mathbf{7 , 8 1 7 6}$ & & $<.0001$ & $<.0001$ & $<.0001$ & $<.0001$ & $<.0001$ \\
$\begin{array}{l}\text { PuR- } \\
\text { MERH }\end{array}$ & $\mathbf{8 , 0 5 7 6}$ & $\mathbf{1 0 , 0 6 4 9}$ & $\mathbf{8 , 6 8 2 1}$ & $\mathbf{6 , 2 3 7 1}$ & $\mathbf{8 , 1 8 2 0}$ & $\mathbf{5 , 0 9 2 2}$ & & $<.0001$ & 0,0033 & 0,0001 & $<.0001$ \\
$\begin{array}{l}\text { RC2- } \\
\text { MECiR }\end{array}$ & 10,5189 & $\mathbf{1 3 , 9 2 3 5}$ & $\mathbf{1 0 , 9 8 4 0}$ & $\mathbf{6 , 0 9 0 6}$ & $\mathbf{8 , 2 2 7 1}$ & $\mathbf{3 , 2 4 4 5}$ & $\mathbf{4 , 0 5 9 2}$ & & $<.0001$ & $<.0001$ & $<.0001$ \\
$\begin{array}{l}\text { RC2- } \\
\text { MERH }\end{array}$ & $\mathbf{2 5 , 9 7 5 4}$ & $\mathbf{2 6 , 0 6 2 4}$ & $\mathbf{1 6 , 4 5 4 1}$ & $\mathbf{1 1 , 4 4 5 5}$ & $\mathbf{1 6 , 5 5 5 5}$ & $\mathbf{7 , 7 7 8 9}$ & 2,0595 & $\mathbf{6 , 6 9 8 9}$ & & $<.0001$ & $<.0001$ \\
$\begin{array}{l}\text { RH10- } \\
\text { MERH }\end{array}$ & $\mathbf{2 1 , 5 6 5 4}$ & $\mathbf{2 1 , 3 6 1 5}$ & $\mathbf{1 6 , 4 5 6 9}$ & $\mathbf{1 0 , 1 4 8 4}$ & $\mathbf{1 3 , 0 6 6 1}$ & $\mathbf{6 , 0 9 2 2}$ & 2,3987 & $\mathbf{5 , 5 6 7 9}$ & $\mathbf{3 , 3 1 7 2}$ & & $<.0001$ \\
$\begin{array}{l}\text { T1- } \\
\text { MECiR }\end{array}$ & 5,0256 & 12,9141 & $\mathbf{6 , 5 1 0 2}$ & 12,2423 & 8,9168 & $\mathbf{1 0 , 6 9 8 9}$ & $\mathbf{8 , 4 3 6 2}$ & $\mathbf{1 1 , 2 9 2 4}$ & $\mathbf{1 8 , 0 0 5 4}$ & $\mathbf{1 6 , 9 1 6 2}$ \\
\hline
\end{tabular}

Tabla 9. Distancias de Procrustes entre pares paisajes rupestres en relación a las modalidades estilísticas (hemimatriz inferior) y P-values de dichas distancias (10000 permutaciones) (hemimatriz superior). Sin efecto del tamaño (free size), considerando solo la forma propiamente dicha. En negrita las distancias significativas.

\begin{tabular}{|c|c|c|c|c|c|c|c|c|c|c|c|}
\hline $\begin{array}{l}\text { Paisajes } \\
\text { rupestres }\end{array}$ & $\begin{array}{c}\text { AP1- } \\
\text { MECiR }\end{array}$ & $\begin{array}{c}\text { AP4- } \\
\text { MECiR }\end{array}$ & $\begin{array}{c}\text { AP16- } \\
\text { MECiR }\end{array}$ & $\begin{array}{c}\text { CC1- } \\
\text { MECiR }\end{array}$ & $\begin{array}{c}\text { CR1- } \\
\text { MECiR }\end{array}$ & $\begin{array}{c}\text { PuR- } \\
\text { MECiR }\end{array}$ & $\begin{array}{c}\text { PuR- } \\
\text { MERH }\end{array}$ & $\begin{array}{c}\text { RC2- } \\
\text { MECiR }\end{array}$ & $\begin{array}{l}\text { RC2- } \\
\text { MERH }\end{array}$ & $\begin{array}{l}\text { RH10- } \\
\text { MERH }\end{array}$ & $\begin{array}{c}\text { T1- } \\
\text { MECiR }\end{array}$ \\
\hline AP1-MECiR & & 0,0230 & 0,0780 & 0,0040 & 0,0410 & 0,0020 & $<.0001$ & $<.0001$ & $<.0001$ & $<.0001$ & 0,0100 \\
\hline AP4-MECiR & 0,0715750 & & $<.0001$ & $<.0001$ & $<.0001$ & $<.0001$ & $<.0001$ & $<.0001$ & $<.0001$ & $<.0001$ & $<.0001$ \\
\hline AP16-MECiR & 0,0590237 & 0,0827175 & & $<.0001$ & 0,0680 & 0,0020 & $<.0001$ & $<.0001$ & $<.0001$ & $<.0001$ & $<.0001$ \\
\hline CC1-MECiR & 0,1025982 & 0,1426294 & 0,0927723 & & 0,0010 & $<.0001$ & $<.0001$ & $<.0001$ & $<.0001$ & $<.0001$ & 0,0030 \\
\hline CR1-MECiR & 0,0828397 & 0,1103291 & 0,0574291 & 0,0799421 & & $<.0001$ & $<.0001$ & $<.0001$ & $<.0001$ & $<.0001$ & 0,0080 \\
\hline PuR-MECiR & 0,1644576 & 0,2016612 & 0,1407123 & 0,1361141 & 0,1107938 & & $<.0001$ & 0,0670 & $<.0001$ & $<.0001$ & 0,0020 \\
\hline PuR-MERH & 0,2491630 & 0,2793479 & 0,2300383 & 0,2261395 & 0,2038894 & 0,1069685 & & $<.0001$ & $<.0001$ & $<.0001$ & $<.0001$ \\
\hline RC2-MECiR & 0,1632086 & 0,2031460 & 0,1390469 & 0,1382955 & 0,1125640 & 0,0442251 & 0,117704 & & $<.0001$ & $<.0001$ & $<.0001$ \\
\hline RC2-MERH & 0,2928982 & 0,3274211 & 0,2710539 & 0,2624982 & 0,2466013 & 0,1457512 & 0,066088 & 0,1522188 & & 0,0030 & $<.0001$ \\
\hline RH10-MERH & 0,3237459 & 0,3532449 & 0,3047363 & 0,29941878 & 0,2763354 & 0,17570933 & 0,0834139 & 0,1893472 & 0,0714705 & & $<.0001$ \\
\hline T1-MECiR & 0,1075586 & 0,1372164 & 0,0961121 & 0,08188950 & 0,0770135 & 0,13217060 & 0,2212031 & 0,1373572 & 0,2630005 & 0,293809 & \\
\hline
\end{tabular}




\section{Discusión}

\subsection{Efecto de variables cualitativas sobre la morfología cuantitativa del camélido inter modalidadestilística.Estandarización, tamaño y técnicas de ejecución.}

Los resultados alcanzados explicitan que las manifestaciones rupestres de camélidos en el sur de Pozuelos presentan una variabilidad moderada en general, pero que claramente permite definir dos grupos de camélidos que reúnen una morfología particular en el espacio plástico. Dichas diferencias se resumen a través del ACP que permite corroborar la existencia de dos conjuntos de camélidos diferenciables correspondiente a la MERH y la MECiR, que presentan variaciones morfológicas en la longitud y ancho del cuerpo; en la postura y ancho de los miembros (anteriores y posteriores); en las alturas a la grupa, a la cruz y subesternal; en la longitud, ancho y disposición del cuello; y en la morfología, tamaño y disposición de la cabeza, cola y oreja.

En la ubicación de las observaciones entre los ejes del CP1 y CP2, que resumen los principales cambios morfológicos, se observa una mayor dispersión de los datos dentro de la MERH en relación a la MECiR, sin embargo, esto no implica que dentro de la MECiR no exista también una considerable variabilidad, aunque más direccionada sobre ciertos rasgos morfológicos en particular (Fig. 5).

Estas variaciones morfológicas identificadas desde los métodos cuantitativos se corresponden con las diferencias identificadas de manera cualitativa en la configuración de los camélidos de ambas modalidades estilísticas (Rodríguez Curletto 2014; Rodríguez Curletto y Angiorama 2016). Dichas morfologías de la MERH y la MECiR se vinculan también a diferencias en las técnicas de ejecución: grabados mediante raspado y picado en surco irregular para la MERH, mientras que para la MECiR se registran las técnicas de pintura (trazo lineal, plano y puntiforme) de colores blanco, negro y rojo (en monocromías y bicromías), como así también grabados (picado plano, en surco, en punto y por abrasión muy uniformes) junto a la presencia de pictograbado (Tabla 3). La mayor dispersión de los datos que presenta sobre los ejes cartesianos la MERH en relación a la MECiR, también es una de las características de los camélidos que habíamos referido desde el análisis estilístico, ya que para la MERH mencionamos una gran complejidad sincrónica (variabilidad y diversidad de morfologías de camélidos), mientras que para la MECiR referimos una marcada estandarización (Rodríguez Curletto 2014).

Estas variaciones entre MERH y MECiR que también fueron cuantificadas a través del ANOVA de Procrustes, indica una diferencia significativa en relación al tamaño del centroide y la forma propiamente dicha (shape), no solo entre modalidades estilísticas sino también en relación a los paisajes rupestres estudiados. Esto permitió conocer las formas promedio de las manifestaciones rupestres de los camélidos de la MERH y la MECiR, y las variaciones morfológicas de cada una en relación a la forma consenso de la muestra total (Fig. 6).

En ambas modalidades estilísticas estas variaciones generales presentan diferentes grados de covariación y correlación entre ellas. A partir del análisis de Regresión Multivariada se observó la existencia de alometría (baja pero significativa), es decir la relación entre la forma propiamente dicha y el tamaño de los elementos, donde una pequeña proporción de las formas puede ser predicha por el tamaño, siendo más elevada para la MECiR y más baja en la MERH (Fig. 7). Al extraer el efecto del tamaño de toda la muestra y junto a las variaciones morfológicas observadas mediante el ACP, se pudo indagar sobre la división de subconjuntos de puntos que definen ciertos caracteres de los camélidos y se encuentran fuertemente integrados entre ellos, pero que al mismo tiempo son relativamente independientes de otros subconjuntos de puntos (módulos). De acuerdo a las variaciones analizadas en el ACP, se pudo comprobar qué tipo de covariación presentaban los caracteres de longitud del cuerpo, altura a la grupa y a la cruz (subconjunto A) en relación a la longitud del cuello, morfología de cabeza, oreja y cola (subconjunto B). Los resultados permiten sostener la hipótesis de Modularidad (baja pero significativa) para la muestra en su totalidad, en la que los subconjuntos A y B pueden ser considerados como módulos distintos con una relativa independencia, aunque resulta interesante destacar que el grado de modularidad varía en relación a las modalidades estilísticas, presentando la MECiR un grado de modularidad menor que la MERH (Fig.8). Asimismo, al incorporar la influencia del tamaño, en ambas modalidades estilísticas se observa la disminución del grado 
de modularidad, es decir que aumenta la covariación entre módulos y por ende aumenta también el grado de dependencia entre los subconjuntos A y B.

Esta situación se comprueba en el grado de integración morfológica observada en la muestra total a través del análisis de Mínimos Cuadrados Parciales que indica una alta y significativa correlación entre los dos subconjuntos A y $\mathrm{B}$, que implica un alto grado de integración morfológica en las manifestaciones de camélidos, es decir, una configuración integrada morfológicamente, donde los cambios pueden ocurrir coordinadamente de manera general, aunque al mismo tiempo se estructura modularmente y por tanto, permite cierta independencia de los subconjuntos definidos (Fig. 9). Asimismo, cuando evaluamos la integración morfológica en relación a las modalidades estilísticas encontramos que los subconjuntos analizados presentan mayor independencia en la MERH (vinculado también al mayor grado de modularidad) en relación a la MECiR, la cual cuenta con un mayor grado de integración morfológica (correlación significativa) en la composición de la figura del camélido. Resulta interesante mencionar que al incorporar el efecto del tamaño aumenta en ambas modalidades el grado de integración morfológica, lo cual se vincula también a la disminución del grado de modularidad como ya mencionamos.

Esto se relaciona con lo presentado en trabajos previos desde el análisis cualitativo donde referimos que en la MECiR se destacaba una gran estandarización técnica y morfológica de la figura del camélido, evidenciando un modo de hacer muy pautado a través de diferentes paisajes rupestres. Dentro de la MECiR, dicha estandarización también se evidencia en otras figuras como la humana, en la composición de motivos como rebaños y caravanas por parte de la figura del camélido, y de este modo una recurrencia y articulación en la conformación de temas caravaneros y pastoriles. En este mismo sentido, la utilización del espacio plástico (direcciones y sentido de ejes horizontal y vertical) por parte de la figura del camélido y su relación con los antropomorfos también es bastante uniforme y repetida, no solo dentro de una misma unidad topográfica sino también entre diferentes paisajes rupestres.

Por otra parte, para la MERH, la situación es totalmente diferente, la figura del camélido no solo presenta una baja estandarización en relación a las técnicas y morfologías, sino que también aparece vinculada a otros cuadrúpedos que por los caracteres morfológicos que presentan no es posible relacionarlos claramente a una especie determinada. Las figuras humanas asociadas, si bien exhiben algunos rasgos generales compartidos, presentan entre ellas una gran variabilidad de formas, disposiciones y técnicas de ejecución (aunque siempre dentro del grabado irregular). Esta baja estandarización también se observa en la gran diversidad de motivos y temas donde la figura del camélido no tiene un rol central como en la MECiR, sino que aparece compartiendo el espacio plástico con variedad de zoomorfos, antropomorfos, geométricos y figuras indeterminadas, destacándose un uso del espacio plástico que puede presentar cantidad y diversidad de sentidos y direcciones de los ejes (vertical y horizontal) dentro de un misma UT (unidad topográfica) (Rodríguez Curletto 2014; Rodríguez Curletto y Angiorama 2016).

\subsection{Efecto de variables cualitativas sobre la morfología cuantitativa del camélido intra modalidad estilística. Paisaje, contexto arqueológico, diacronía, técnicas de ejecución y distancias geográficas.}

Cuando comparamos las medias grupales de la MERH y la MECiR, y de los diez paisajes rupestres en relación a dichas modalidades, observamos que las distancias de Mahalanobis y Procrustes son significativas para ambas modalidades, puede observarse claramente la discriminación más efectiva de las mismas sobre la VC1 y cuáles son las formas más frecuentes dentro de cada modalidad (Fig. 10). Al abordar dichas diferencias a través del Análisis Discriminante, se pudo comprobar la correcta asignación de los diferentes camélidos analizados a cada modalidad estilística definida, y por tanto una muy baja reclasificación de camélidos en una modalidad distinta a la de su pertenencia original, es decir que aquella clasificación a priori realizada a través del estudio cualitativo de la forma, alcanza un valor de acierto considerable al evaluarlo de manera cuantitativa.

Por otra parte, al analizar estas distancias entre paisajes rupestres, encontramos que más allá de las distancias significativas entre paisajes de diferente modalidad estilística, lo cual era esperable de acuerdo a los resultados hasta aquí presentados, se observan además distancias significativas entre paisajes rupestres 
vinculados a la misma modalidad estilística. Esta situación lleva a pensar en la influencia de otras variables que exceden a la definición de la morfología de los camélidos en sí misma y que deberán ser analizadas con detalle en estudios futuros. Estas variables corresponderían a: las propiedades de los diferentes contextos arqueológicos y prácticas asociadas a cada paisaje rupestre; las diferencias en las técnicas de ejecución aún dentro de la misma modalidad estilística; las diferencias cronológicas relativas (diacronía) entre técnicas y eventos de ejecución intra e inter paisajes rupestres y finalmente, las distancias geográficas entre dichos paisajes rupestres. Todas estas variables podrían estar actuando por separado o en conjunto sobre los diversos paisajes.

Las distancias significativas se evidencian tanto en la MERH, donde los modos de hacer los grabados son muy irregulares intra e inter paisaje rupestre, así como también en la ME$\mathrm{CiR}$, que si bien sus modos de hacer intra sitio son más homogéneos, aun así la influencia de las variables anteriormente mencionadas (variedad de técnicas de ejecución, distancia geográfica, diferencias cronológicas relativas y de los contextos arqueológicos asociados), estarían jugando un rol importante en las distancias morfológicas ${ }^{13}$ entre los camélidos de sus diferentes paisajes rupestres.

$\mathrm{Si}$ consideramos para la MECiR las distancias de Mahalanobis, encontramos que los camélidos de los paisajes rupestres de AP1, AP4, AP16, CiR1, CC1, y T1 no presentan diferencias significativas entre sí, siendo el AP1 el que no presenta distancias significativas con ninguno de los paisajes de la MECiR. En sentido opuesto, el RC2-MECiR ${ }^{14}$ presenta distancias significativas con todos los paisajes de la MECiR, con excepción de AP1, mientras que el PuR-MECiR presenta distancias significativas con la mayoría de estos paisajes excepto con AP1 y CC1. Estas diferencias pueden deberse a la influencia de la técnica de ejecución en la morfología general de los camélidos. Recordemos que mientras en AP1, AP4, AP16, CC1 y T1 los camélidos han sido realizados solo mediante la técnica de pintura, en RC2MECiR y PuR-MECiR la gran mayoría han sido ejecutados mediante la técnica de grabado y pictograbado. Algo similar ocurre si consideramos dentro de la MECiR las distancias de Procrustes en cuanto a las distancias significativas de PuR-MECiR y RC2-MECiR del resto de los paisajes de dicha modalidad, solo que en este caso el PuR y RC2 no presentan distancias significativas entre sí, reafirmando el vínculo entre ellos en relación a las técnicas de ejecución compartidas.

Más allá de la influencia de la técnica de ejecución también es posible considerar una influencia temporal en la que diferentes morfologías y técnicas de ejecución podrían vincularse a diferencias cronológicas relativas dentro de la MECiR que aún estamos indagando. Estos cambios técnicos, por ejemplo, la combinación de colores (monocromías o bicromías), estarían acompañados por cambios leves en las morfologías de los camélidos. Ejemplo de ello puede ser la ausencia de distancias significativas entre $\mathrm{CC} 1$, AP1 y T1 que presentan camélidos monocromáticos (rojo), y por otra parte, las distancias significativas entre $\mathrm{CC} 1$ y $\mathrm{T} 1$ en relación a CiR1 y AP16, estos últimos con camélidos bicromáticos (blanco y rojo) en su mayoría. La influencia de la variable temporal también se sostiene al evaluar el caso de AP1, que es el que presenta menor distancia (Procrustes y Mahalanobis) con todos los paisajes de la MECiR, situación que se fundamenta en que AP1 cuenta claramente con dos eventos de ejecución diferentes (Conjunto Técnico-cromático 1 y Conjunto Técnico-cromático 2) que justamente sintetiza las variaciones morfológicas de diferentes cronologías relativas, lo cual fundamentaría su menor distancia en relación a paisajes de la MECiR que pueden presentar vínculos con algunos de estos dos eventos de AP1. En el mismo sentido, cierta influencia de la variable temporal puede interpretarse en relación al PuR-MECiR y RC2-MECiR que como ya mencionamos se diferencian de la mayoría de los paisajes rupestres de la MECiR, posiblemente debido también a la gran cantidad de eventos de ejecución que presentan ambos paisajes, situación que les confiere una complejidad diacrónica que necesariamente puede influir en mayor variabilidad de morfologías, y por tanto aumentar las distancias morfológicas con el resto de los paisajes. En el caso de RC2, por ejemplo, presenta 49 Conjuntos Técnicos y Técnicos-cromáticos vinculados a la MECiR.

Por otra parte, si bien no se registra en todos los casos, es posible observar que, en la gran mayoría de los paisajes rupestres de la MECiR, las distancias morfológicas aumentan de modo directamente proporcional a las distancias espaciales entre los paisajes rupestres siendo AP1 el que de alguna manera constituye un punto de inicio a partir del cual pueden 
trazarse los valores ascendentes de las distancias morfológicas en relación a las distancias geográficas relativas. Asimismo, cabe destacar que entre modalidades estilísticas la distancia espacial no tiene efecto ya que aun compartiendo el mismo paisaje rupestre como ocurre en el PuR y RC2, ambas modalidades MERH y la MECiR presentan distancias significativas tanto de Mahalanobis como de Procrustes.

Por otra parte, algunas de estas distancias intra modalidad estilística, podrían ser explicadas por la influencia de los contextos arqueológicos asociados, los temas en los que participan los camélidos y las diferentes prácticas a las que estarían vinculados dichos paisajes. Ejemplo de ello, sería AP1 que cuenta con un repertorio temático y un contexto arqueológico vinculado al caravaneo, siendo interpretado como realero o jarana ubicado en un punto coyuntural para el tráfico de la microrregión que conecta el interior de la cuenca de Pozuelos con el oeste (Cuenca del río Orosmayo, lagunas altoandinas y región atacameña), y que tal como hemos mencionado no presenta distancias morfológicas significativas con ningún otro paisaje de la MECiR. Esta situación para este "lugar" vinculado al tráfico caravanero y tan relevante en el entramado de las conexiones entre el interior y el exterior de la cuenca, puede ser interpretada como la necesidad de compartir o resumir una configuración del camélido que pueda ser vinculada fácilmente al resto de los paisajes rupestres del interior de la cuenca. Por tanto, las morfologías de los camélidos de AP1 no presentan distancias significativas ni con una base residencial relacionada a prácticas agro-pastoriles como $\mathrm{T} 1$, ni a un asentamiento eje como el PuR, ni tampoco a un lugar ritual caravanero (ruta de tránsito) y base residencial como es el caso de $\mathrm{CC} 1$.

Al considerar para la MERH la distancia de Mahalanobis, también observamos diferencias significativas entre los paisajes rupestres vinculados a esta modalidad. Por una parte, RH10 y RC2-MERH se diferencian entre sí, aunque con valores muy pequeños, pero al mismo tiempo, ambos son similares al PuR-MERH. Si evaluamos las distancias de Procrustes se observa que mientras el PuR-MERH presenta diferencias significativas con todos los paisajes, incluidos RH10 y RC2-MERH (aunque con valores bajos entre sí), estos solamente presentan relaciones entre sí y totalmente aislados del resto. Esto puede deberse a la variabilidad interna que presenta la MERH en general, como hemos referido anteriormente, lo cual se refleja en la ambigüedad observada de acuerdo a las diferentes distancias consideradas (Procrustes y Mahalanobis). Sin embargo, es importante mencionar que dichas distancias presentan los valores más bajos dentro de las distancias significativas, y por tanto estas diferencias siguen siendo menores que las registradas entre modalidades diferentes.

\section{Conclusiones}

Las relaciones entre los "modos de hacer" la figura del camélido, cronología y paisajes socioculturales ha sido una discusión constante en las investigaciones sobre el arte rupestre de los Andes del Sur en general, y del NOA en particular (Aschero 2000; Berenguer 2004; Troncoso 2012; Martel 2010; entre otros). Los camélidos en el arte rupestre del sur de Pozuelos (Puna Argentina) constituyen una fuente muy valiosa para abordar diferentes aspectos del contexto socio-cultural que los generó.

$\mathrm{Su}$ alta frecuencia y relativa diversidad en los diferentes paisajes vinculados a momentos de los Desarrollos Regionales (ca. 900-1430 DC), nos llevó a preguntarnos sobre la manera en que la configuración de los mismos es perfomada en diferentes motivos, temas, unidades topográficas y paisajes. Los estudios cualitativos realizados previamente en el arte rupestres del sur de Pozuelos, nos permitieron definir, desde el estudio contextual, estilístico y del paisaje, las dos modalidades estilísticas MERH y MECiR (Rodríguez Curletto 2014; Rodríguez Curletto y Angiorama 2016). Esta primera aproximación constituyó la base sobre la cual este trabajo abordó cuantitativamente la figura del camélido, a través de la Morfometría Geométrica en relación a dichas modalidades estilísticas y a los paisajes rupestres del sur de Pozuelos.

Los diferentes métodos estadísticos multivariados aplicados a la morfología de los camélidos permitieron explorar la diversidad y las variaciones morfológicas en la muestra estudiada, cuantificar y evaluar la magnitud de dichas variaciones, así como también analizar la presencia de covariación y correlación entre las variables que influyen en la conformación de la figura del camélido.

De esta manera, desde la Morfometría Geométrica se pudo corroborar y sustentar cuantitativamente aquellos caracteres y configuraciones en la figura del camélido planteados desde el estudio estilístico. A través del ACP y el ANOVA de Procrustes se corroboró 
la existencia de diferencias morfológicas significativas entre dos conjuntos de camélidos correspondientes a la MERH y la MECiR. Esto permitió conocer las formas promedio de la figura del camélido en cada modalidad, las variaciones morfológicas de cada una en relación a la forma consenso de la muestra total, y también conocer en que partes y cómo se daban esas diferencias en la composición de dicha figura.

En ambas modalidades las variaciones morfológicas se observan en la longitud y ancho del cuerpo; en la postura y ancho de miembros (anteriores y posteriores); en las alturas a la grupa, a la cruz y subesternal; en la longitud, ancho y disposición del cuello; y en la morfología, tamaño y disposición de la cabeza, cola y oreja.

A través de la técnica de Regresión Multivariada, se sustenta la existencia de alometría que constituye una de las causas de los cambios morfológicos del camélido en ambas modalidades estilísticas, siendo mayor su incidencia en la MECiR.

Los análisis cualitativos junto a los resultados obtenidos en la Hipótesis de Modularidad, Integración Morfológica y Mínimos Cuadrados Parciales; apoyan lo planteado por Aschero (2000) acerca de la mayor estandarización de algunas figuras en el área de circumpuna para el período del ca. 900 a 1500 DC. La estandarización de las morfologías de la figura del camélido en los "modos de hacer" se registra en ambas modalidades estilísticas, siendo menor en la MERH en relación a la MECiR, esta última con un modo de hacer muy pautado que ya había sido vislumbrado desde el estudio cualititivo (Rodríguez Curletto 2014; Rodríguez Curletto y Angiorama 2016)

A través del Análisis de Variantes Canónicas y el Análisis de la Función Discriminante se observó que las distancias de Mahalanobis y Procrustes son significativas para ambas modalidades estilísticas. Se pudo observar claramente la discriminación más efectiva de las mismas y cuáles son las formas más frecuentes dentro de cada modalidad. Al abordar dichas diferencias a través del Análisis de la Función Discriminante, se pudo comprobar en una alta proporción, la correcta asignación de los diferentes camélidos a cada modalidad estilística definida.

Es interesante destacar, que más allá de las mencionadas diferencias (alometría, modularidad, integración morfológica) y distancias morfológicas significativas entre la MERH y la MECiR; los análisis aplicados permitieron también, identificar diferencias y distancias morfológicas significativas en la figura del camélido entre distintos paisajes rupestres, más allá de su pertenencia a una misma modalidad estilística.

Consideramos que estas diferencias podrían ser explicadas por el efecto de otras cuatro variables que interactúan en diferentes grados sobre la morfología del camélido. Estas variables, que deberán ser indagadas con mayor profundidad en trabajos futuros, corresponden a: las propiedades de los contextos arqueológicos y prácticas asociadas a cada paisaje rupestre; las diferencias en las técnicas de ejecución aún dentro de la misma modalidad estilística; las diferencias cronológicas relativas (diacronía) entre técnicas y eventos de ejecución intra e inter paisaje rupestre y finalmente; la distancia geográfica entre dichos paisajes.

A partir de todo lo expuesto, se observa que los resultados obtenidos de la Morfometría Geométrica, fundamentan y profundizan notablemente las interpretaciones alcanzadas en estudios cualitativos realizados previamente para la MECiR y la MERH (Rodríguez Curletto 2014; Rodríguez Curletto y Angiorama 2016, Rodríguez Curletto et al. 2019). Ante esta situación, es importante destacar el potencial de integrar métodos cuantitativos y cualitativos para abordar la complejidad de la figura del camélido en paisajes rupestres vinculados a diferentes prácticas y contextos arqueológicos. En este sentido, consideramos que el trabajo aquí presentado constituye un punto de partida, no solo para preguntas específicas sobre los camélidos en el arte rupestre del sur de Pozuelos, sino también para extender este tipo de enfoque a escala regional ${ }^{15}$ en los Andes del Sur, que nos permita abordar y discutir estas problemáticas, procesos y paisajes socioculturales, con una mirada macro-regional. 


\section{Bibliografía}

Adams, D. (1999): Methods for shape analysis of landmark data from articulated structures. Evolutionary Ecological Research, 1: 959-970.

Angiorama, C. (2011): La ocupación del espacio en el Sur de Pozuelos (Jujuy) durante tiempos prehispánicos y coloniales. Estudios Sociales del NOA 11:125-142.

Aschero, C. (2000): Figuras humanas, camélidos y espacio en la interacción circumpuneña. Arte en las Rocas: Arte Rupestre, Menhires y Piedras de Colores en Argentina (M. Podestá, M.; de Hoyos, eds.), Sociedad Argentina de Antropología, Buenos Aires: 15-44.

Benítez, H.; Püschel, T. (2014): Modelando la varianza de la forma: morfometría geométrica aplicaciones en biología evolutiva. Int. J. Morphol., 32(3):998-1008.

Berenguer, J. (2004): Caravanas, Interacción y Cambio en el Desierto de Atacama. Sirawi Ediciones, Santiago de Chile.

Bookstein, F. (1995): The morphometric synthesis for landmarks and edge-elements in images. Terra Nova, 7: 393-407.

Bookstein, F. (1996): Biometrics, biomathematics and the morphometric synthesis. Bulletin of Mathematical Biology, 58(2): 313-365.

Bookstein, F. (1996-1997): Landmarks methods for form without landmarks: Morphometrics of group differences in outline shape. Medical Image analysis, 1(3): 225-243.

Bookstein, F.; Gunz, P.; Mitteroecker, P.; Prossinger, H.; Schaefer, K.; Seidler, H. (2003): Cranial integration in Homo: singular warps analysis of the midsagittal plane in ontogeny and evolution. Journal of Human Evolution, 44: 167-187.

Cardillo, M. (2010): Some applications of Geometric Morphometrics to Archaeology. Morphometrics for nonmorphometricians (A. Elewa, ed.), Springer, Verlag- Berlin- Heidelberg. 325-355.

Charlin, J.; Hernández Llosas, M. (2016): Morfometría geométrica y representaciones rupestres: explorando las aplicaciones de los métodos basados en landmarks. Arqueología 22(1):103-125.

Escoufier, Y. (1973): Le traitement des variables vectorielles. Biometrics 29:751-760.

Goodall, C. (1991): Procrustes methods in the statistical analysis of shape. Journal of the Royal Statistical Society, 53:285-339.

Gunz, P.; Mitteroecker, P. (2013): Semilandmarks: a method for quantifying curves and surfaces. Hystrix, the Italian Journal of Mammalogy, 24(1): 103-106.

Ingold, T. (2000): The Perception of the Environment. Essays on livelihood, Dwelling and Skill. Routledge, London.

Johnson, D. (2000): Métodos multivariados aplicados al análisis de datos. Alfaomega, México.

Klingenberg C.; McIntyre G. (1998): Geometric morphometrics of developmental instability: analyzing patterns of fluctuating asymmetry with Procrustes methods. Evolution 52: 1363-1375.

Klingenberg, C.; Monteiro, L. (2005): Distances and Directions in Multidimensional Shape Spaces: Implications for Morphometric Applications. Systematic Biology, 54(4): 678-688. doi. org/10.1080/10635150590947258.

Klingenberg, C. (2009): Morphometric integration and modularity in configurations of landmarks: Tools for evaluating a-priori hypotheses. Evolution \& Development 11:405-421.

Klingenberg, C. (2011): MorphoJ: an integrated software package for geometric morphometrics. Molecular Ecology Resources, 11:353-357. doi:10.1111/j.1755-0998.2010.02924.x

Martel, A. (2010): Arte rupestre de pastores y caravaneros. Estudio contextual de las representaciones rupestres durante el periodo Agroalfarero Tardio (900-1480 DC) en el Noroeste argentino. Tesis Doctoral inédita. FFyL (UBA), Buenos Aires.

Mitteroecker, P.; Gunz, P. (2009): Advances in Geometric Morphometrics. Evolutionary Biology, 36: 235-247.

Nelson, E.; Hall, J.; Randolph-Quinney, P.; Sinclair, A. (2017): Beyond size: The potential of a geometric morphometric analysis of shape and form for the assessment of sex in hand stencils in rock art. Journal of Archaeological Science, 78:202-213.

Nielsen, A. (2007): Armas significantes: tramas culturales, guerra y cambio social en el Sur Andino Prehispánico. Boletín del Museo Chileno de Arte Precolombino 12 (1): 9-41.

Pérez, I.; Cardillo, M.; González, P. (2009): Artificial cranial deformations in South America: a quantitative approach to cultural variation. Arqueología y Evolución. Teoría, metodología y casos de estudio (López, G.; Cardillo, M. eds.), Editorial sb, Buenos Aires: 87-108. 
Quispe Coaquira, J.; Apaza Zúñiga, E.; Ibáñez Quispe, V.; Villalta Ticona, R.; Calsín Calsín, B.; Vilca Castro, C. (2015): Caracterización morfológica índices corporales de llamas (Lama glama) Ch'acu y k'ara de la puna húmeda de la Región Puno. Journal of High Andean Research, 17 (2):183-192.

Rodríguez Curletto, S. (2014): Paisajes y Estilos del Arte Rupestre en la Cuenca Sur de Pozuelos. Tesis de Grado inédita. FCNeIML (UNT), Tucumán.

Rodríguez Curletto, S., Angiorama, C. (2016): El arte rupestre del sur de la cuenca de Pozuelos (900-1535 DC). Boletín del Museo Chileno de Arte Precolombino 21 (2): 25-46.

Rodríguez Curletto, S., Angiorama, C. (2017): Lesiones traumáticas en cráneos humanos. Aproximación a la violencia interpersonal durante el Tardío en la Puna de Jujuy, Argentina. Libro de resúmenes de las XIII Jornadas Nacionales de Antropología Biológica, Necochea, Argentina: 162.

Rodríguez Curletto, S.; Lauricella, M.; Angiorama, C. (2019): Paisajes rupestres vinculados a la trashumancia y al caravaneo durante los Desarrollos Regionales (900-1430 DC) en el sur de Pozuelos (Puna de Jujuy, Argentina). Chungara, 51(4): http://dx.doi.org/10.4067/S0717-73562019005001901

Rohlf, J.; Slice, D. (1990): Extensions of the Procrustes method for the optimal superimposition of landmarks. Systematic Zoology, 39 (1): 40-59.

Rohlf, J.; Marcus, L. (1993): A revolution in Morphometrics. Trends in Ecology and Evolution, 8: 129-132.

Rohlf, J. (2008): tpsUtility version 1.40. Department of Ecology and Evolution, State University. Stony Brook, New York.

Rohlf, J. (2013): tpsDig2 version 2.17. Department of Ecology and Evolution, State University, Stony Brook, New York.

Slice, D. (2007): Geometric morphometrics. Annual Review of Anthropology, 36: 261-281.

Slice, D.; Bookstein, F.; Marcus, L.; Rolhf, J. (2009): A Glossary for Geometric Morphometrics. http://life. bio.sunysb.edu/morph/glossary/gloss $1 . \mathrm{html}$

Tilley, C. (2004): The Materiality of Stone: Explorations in Landscape Phenomenology. Berg, Oxford.

Torcida, S.; Perez, I. (2012): Análisis de Procrustes y el estudio de la variación morfológica. Revista Argentina de Antropología Biológica, 14 (1): 131-141.

Troncoso, A. (2012): Arte rupestre y camélidos en el Norte Semiárido de Chile: una discusión desde el valle de Choapa. Boletín del Museo Chileno de Arte Precolombino, 17 (1): 75-93, Santiago de Chile.

Tuco Cano, E. (2009): Zoometric measures in llamas (Lama glama) of rural families in the Tolar and Pajonal natural grazing fields of the municipality Santiago de Machaca - department of La Paz. All Theses and Dissertations. 5440. https://scholarsarchive.byu.edu/etd/5440

Zelditch, M.; Swiderski, D.; Sheets, H.; David, H. (2014): A Practical Companion for Geometric Morphometrics for Biologists: Running analyses in freely-available software. http://booksite.elsevier. com/9780123869036/content/Workbook.pdf

\section{Notas}

1. Agradecemos a cada uno de los habitantes del sur de Pozuelos (Puna de Jujuy, Argentina) por permitirnos trabajar en su territorio por más de una década, y a todos los miembros del equipo que participaron en las tareas de campo realizadas en el área de estudio. Las investigaciones fueron financiadas mediante subsidios PICT (FONCyT), PIP (CONICET) y PIUNT (Universidad Nacional de Tucumán). 2. Las Unidades Topográficas (UT) son definidas a partir de la orientación y topografía de la superficie rocosa, y por la configuración distribucional de los motivos.

3. Se refiere a puntos que pueden ser localizados repetidamente por equivalencia morfológica o funcional en todos los camélidos (Charlin y Hernandez Llosas 2016).

4. La unidad de análisis aquí es la figura del camélido (elemento), y para la MG se requiere de una imagen por elemento analizado, por tanto, se procesaron en total 330 imágenes correspondientes a 330 elementos.

5. Se incorporan las regiones zoométricas para que sirvan como puntos estandarizados de extrapolación en el análisis de la figura del camélido en el arte rupestre, y de ese modo facilitar la comprensión de sus cambios morfológicos entre regiones, contextos y cronologías.

6. Siguiendo a Charlin y Hernandez Llosas (2016) nos referirnos a la "forma propiamente dicha" como equivalente a shape, y a forma (form en inglés) como la suma de shape + size. Las coordenadas de forma de Procrustes refieren justamente a shape. 
7. La implementación del Análisis de la Función Discriminante en MorphoJ utiliza la regla de clasificación de Fisher, que establece el punto de corte en un valor de cero.

8. Los Valores de P en estas pruebas usan los dos tipos de distancias (Procrustes y Mahalanobis) y pueden variar entre ellas para una misma comparación porque mientras la distancia de Procrustes mide la distancia euclidiana entre conjuntos de coordenadas de forma, esto es, la raíz cuadrada de la suma de las diferencias de las coordenadas al cuadrado (Mitteroecker y Gunz 2009), la distancia de Mahalanobis es medida entre puntos considerando la varianza y la correlación de las variables, es decir mide las diferencias entre los grupos en relación con la variación dentro del grupo (Klingenberg y Monteiro 2005).

9. El criterio de Pillai es un estadístico para contrastar la hipótesis nula de igualdad de vectores de medias. Dicho criterio utiliza todas las raíces características y se aproxima a ellas a partir del estadístico F (compara la variabilidad producto de las diferencias entre grupos con la debida a las diferencias dentro de los grupos), de este modo cuanto mayor sea el valor de F y menor su significación, habrá más probabilidad de que existan diferencias significativas entre los grupos.

10. Para este análisis fue utilizado el log centroid size en lugar del centroid size con el fin de linearizar la relación entre ambas variables y mejorar sus propiedades estadísticas (Zelditch et al. 2014).

11. El Coeficiente de RV (Escoufier 1973) es una medida de covariación entre dos conjuntos de puntos de referencia, es decir cuantifica la fuerza de asociación entre dos conjuntos de variables (matrices de datos). En MG estimar el coeficiente RV (generalización multivariable del coeficiente de correlación al cuadrado) sirve para analizar la independencia relativa de los hipotéticos módulos. Entre los módulos evaluados un $\mathrm{RV}=0$ implica una baja dependencia (menor covariación), mientras que un $\mathrm{RV}=1$ implica una fuerte dependencia (mayor covariación) (Klingenberg 2009). 12. El análisis PLS de MorphoJ permite examinar la covariación entre bloques de puntos de referencia dentro de una única configuración. Dicho análisis considera toda la covariación entre bloques (incluido el componente de los tamaños relativos y las posiciones) (Klingenberg 2011). 13. Un factor importante a considerar en la evaluación de las distancias obtenidas en las tablas 8 y 9 corresponde al número de observaciones presentes en cada paisaje rupestre, ya que los resultados pueden verse afectados por la desigualdad en la cantidad de elementos de cada paisaje rupestre. Por tal motivo, dichas interpretaciones deben ser tomadas a modo de referencia, con la intención de ser abordadas con mayor precisión sumando mayor cantidad de observaciones en algunos de estos paisajes rupestres.

14. RC2-MECiR refiere a los camélidos de Río Candado 2 (RC2) adscribibles a la MECiR, dicha lógica se repite en las siguientes asociaciones de Paisaje rupestre-Modalidad.

15. En trabajos previos, hemos presentado evidencias de la transposición de la MERH y la MECiR a nivel regional. La MECiR se transpone hacia paisajes del sur de la puna de Jujuy (Casabindo, Doncellas, Coranzulí, Susques, Barrancas, y otros sectores de la cuenca de GuayatayocMiraflores), hacia el norte del Valle Calchaquí, y en lugares localizados al este de nuestra área de estudio (Tres Cruces, Inca Cueva y la Quebrada de Humahuaca). Por otra parte, la MERH, se transpone desde paisajes ubicados en el sector norte de nuestra área de estudio hacia la puna septentrional de Jujuy, y hacia el sur de Bolivia (Rodríguez Curletto et al. 2019). 